\title{
The Discrete Galerkin Method for Integral Equations
}

\author{
By Kendall Atkinson* and Alex Bogomolny
}

\begin{abstract}
A general theory is given for discretized versions of the Galerkin method for solving Fredholm integral equations of the second kind. The discretized Galerkin method is obtained from using numerical integration to evaluate the integrals occurring in the Galerkin method. The theoretical framework that is given parallels that of the regular Galerkin method, including the error analysis of the superconvergence of the iterated Galerkin and discrete Galerkin solutions. In some cases, the iterated discrete Galerkin solution is shown to coincide with the Nyström solution with the same numerical integration method. The paper concludes with applications to finite element Galerkin methods.
\end{abstract}

1. Introduction. Consider the numerical solution of the Fredholm integral equation of the second kind,

$$
x(s)-\int_{D} K(s, t) x(t) d \sigma(t)=y(s), \quad s \in D
$$

In this paper we will define and analyze the discrete Galerkin method for the numerical solution of (1.1). The Galerkin method is a well-known procedure for the approximate solution of this equation (e.g., see [5, p. 62]); and the discrete Galerkin method results when the integrations of the Galerkin method are evaluated numerically. Before giving a more precise definition of the discrete Galerkin method, we review results for the Galerkin method.

In Eq. (1.1), the region $D$ is to be a closed subset of $\mathbf{R}^{m}$, some $m \geqslant 1$; and the dimension of $D$ can be less than $m$, for example, if $D$ is a surface in $\mathbf{R}^{3}$. For the discrete Galerkin method, we will assume that $K(s, t)$ is continuous for $s, t \in D$, although that is not necessary for the discussion of Galerkin's method given below. The equation (1.1) is written symbolically as

$$
(I-\mathscr{K}) x=y
$$

with the integral operator assumed to be a compact operator from $L^{2}(D)$ to $L^{2}(D)$ and from $L^{\infty}(D)$ to $C(D)$. Further, it is assumed that (1.1) is uniquely solvable in $\mathscr{X}$ for all $y \in \mathscr{X}$, for both $\mathscr{X}=C(D)$ and $\mathscr{X}=L^{2}(D)$. Additional assumptions on $D$ and $K(s, t)$ will be given as they are needed in the applications presented later in the paper. Generally $y \in C(D)$, and this will imply $x \in C(D)$.

Received July 5, 1985; revised March 14, 1986.

1980 Mathematics Subject Classification. Primary 65R20.

*Supported in part by NSF grant DMS-8403131. 
The Galerkin method for solving (1.2) is defined as follows. Let $\left\{S_{h}: h>0\right\}$ denote a sequence of finite-dimensional approximating subspaces of both $L^{2}(D)$ and $C(D)$, and let $P_{h}$ denote the orthogonal projection of $L^{2}(D)$ onto $S_{h}$. Then to approximate (1.2), solve

$$
\left(I-P_{h} \mathscr{K}\right) x_{h}=P_{h} y, \quad x_{h} \in L^{2}(D) ;
$$

or equivalently, pick $x_{h} \in S_{h}$ such that

$$
\left((I-\mathscr{K}) x_{h}, \psi\right)=(y, \psi), \quad \text { all } \psi \in S_{h},
$$

using the inner product of $L^{2}(D)$. This is called the Galerkin method for (1.2), relative to the subspace $S_{h}$. In addition, define the iterated Galerkin solution by

$$
x_{h}^{*}=y+\mathscr{K} x_{h} .
$$

The error analysis of $x_{h}$ and $x_{h}^{*}$ is well known, both in $L^{2}(D)$ and $C(D)$. For a simple error analysis of $x_{h}$ in $L^{2}(D)$, see [5, p. 62]; for more general error analyses of $x_{h}$ and $x_{h}^{*}$, in both $L^{2}(D)$ and $C(D)$, see [12], [13], [15], [28], [29].

The error analysis of $x_{h}$ and $x_{h}^{*}$, whether in $L^{2}(D)$ or $C(D)$, usually depends on showing that

$$
\left\|\mathscr{K}-P_{h} \mathscr{K}\right\| \rightarrow 0 \quad \text { as } h \rightarrow 0
$$

with the norm dependent on which space $\mathscr{X}=L^{2}(D)$ or $C(D)$ is being considered. If

$$
P_{h} x \rightarrow x \text { as } h \rightarrow 0 \text {, all } x \in \mathscr{X},
$$

then (1.6) follows from the compactness of $\mathscr{K}$ on $\mathscr{X}$; and generally we will be assuming (1.7) for both choices of $\mathscr{X}$. From (1.6) and the assumed existence of $(I-\mathscr{K})^{-1}$ on $\mathscr{X}$, it follows that $\left(I-P_{h} \mathscr{K}\right)^{-1}$ exists and is uniformly bounded for all sufficiently small values of $h$. Furthermore,

$$
\begin{gathered}
x-x_{h}=\left(I-P_{h} \mathscr{K}\right)^{-1}\left(x-P_{h} x\right), \\
\left\|x-x_{h}\right\| \leqslant\left\|\left(I-P_{h} \mathscr{K}\right)^{-1}\right\|\left\|x-P_{h} x\right\| .
\end{gathered}
$$

Together with (1.7), this shows convergence, along with the rate of convergence. The value of $\left\|x-P_{h} x\right\|$ will depend on both $S_{h}$ and the smoothness of the unknown solution $x$.

For the iterated Galerkin solution $x_{h}^{*}$, it is straightforward to show that

$$
\left(I-\mathscr{K} P_{h}\right) x_{h}^{*}=y
$$

and that

$$
P_{h} x_{h}^{*}=x_{h} .
$$

Using an error analysis for $x_{h}^{*}$, one can also be given for $x_{h}$ as follows:

$$
\begin{gathered}
x-x_{h}=x-P_{h} x_{h}^{*}=\left[x-P_{h} x\right]+P_{h}\left[x-x_{h}^{*}\right], \\
\left\|x-x_{h}\right\| \leqslant\left\|x-P_{h} x\right\|+\left\|P_{h}\right\|\left\|x-x_{h}^{*}\right\| .
\end{gathered}
$$

The analysis of $(1.10)$ in $L^{2}(D)$ hinges on the fact that with the corresponding operator norm,

$$
\left\|\mathscr{K}-\mathscr{K} P_{h}\right\|=\left\|\mathscr{K}^{*}-P_{h} \mathscr{K}^{*}\right\| \rightarrow 0 \quad \text { as } h \rightarrow 0 .
$$

The compactness of $\mathscr{K}$ implies the same for $\mathscr{K}^{*}$, and then the assumption (1.7) implies $\left\|\mathscr{K}^{*}-P_{h} \mathscr{K}^{*}\right\| \rightarrow 0$. Derivation of (1.13) employs the fact that $P_{h}$ is an orthogonal projection in $L^{2}(D)$. When $P_{h}$ is not orthogonal, as on $\mathscr{X}=C(D)$, one 
uses ad hoc methods to establish superconvergence; for example, see [15]. It is the purpose of this note to introduce a formalism that allows the derivation of similar estimates for a wide class of discrete Galerkin methods.

Using (1.13), a straightforward stability and convergence analysis can be given for (1.10). In fact, (1.10) is a degenerate kernel method if the application of (1.10) to (1.1) is examined in more detail. The analysis of (1.10) generalizes to $C(D)$. For this, see [12], [13], [15], [28], [29].

For (1.10),

$$
x-x_{h}^{*}=\left(I-\mathscr{K} P_{h}\right)^{-1} \mathscr{K}\left(I-P_{h}\right) x .
$$

Since $I-P_{h}$ is a projection, $\left(I-P_{h}\right)=\left(I-P_{h}\right)^{2}$ and

$$
\left\|x-x_{h}^{*}\right\| \leqslant\left\|\left(I-\mathscr{K} P_{h}\right)^{-1}\right\|\left\|\mathscr{K}\left(I-P_{h}\right)\right\|\left\|\left(I-P_{h}\right) x\right\| .
$$

Using (1.13) for the case $\mathscr{X}=L^{2}(D),(1.15)$ shows that $x_{h}^{*}$ converges to $x$ more rapidly than does $x_{h}$. A similar result can be shown in the space $C(D)$; see the papers cited above. This more rapid convergence is called 'superconvergence'.

In practice, the integrals in (1.4) and (1.5) are not computed exactly, which leads to the discrete and discrete iterated Galerkin methods, respectively. We shall introduce these discrete methods in the next section, initially posing them in a matrix algebra framework. It is shown that in many important cases, the iterated discrete Galerkin method is exactly the same as the direct application of the numerical integration method to (1.1), yielding a Nyström method. Section 3 contains some applications and implications of this result. In Sections 4, 5, and 6 we introduce a functional analysis framework for discrete Galerkin methods, a framework that is a 'discrete analogue' of the analyses given above for the regular Galerkin method. Unlike the previous analyses of the discrete Galerkin method by [12, Chapter 3], [23], [30], our approach yields convergence results for the discrete methods directly, without referring to the convergence of their continuous analogues. Albeit more restrictive, our approach yields more general results where applicable. For reasons of space, Section 6 is placed separately at the end of the issue, in photo-offset form. Section 7 contains applications of this method to several classes of problems.

This paper is devoted to the nonhomogeneous equation (1.1), but the results generalize to the numerical solution of the associated eigenvalue problem. The approximating operators in Section 5 are shown to be a collectively compact family. This means that the standard analyses of the numerical eigenvalue problem can be used, for example, [1], [3], [4], [14, Chapiers 5-7], and [25]; and these results can be combined with the techniques of this paper to give an analysis of the discrete Galerkin method for solving the eigenvalue problem. Future papers will discuss the eigenvalue problem, iterative variants to solve the associated linear systems, and applications to Galerkin methods for nonlinear equations.

Discrete Galerkin methods for boundary value problems have also been analyzed previously. An important early paper is [20]; and an analysis of a discrete leastsquares method is given in [2]. Although their results are related to those given here, our schema is more general and is not as restricted in the properties of the operators, the approximating subspaces, and the numerical integration scheme. 
2. The Discrete Galerkin Method. The Galerkin equation (1.3)-(1.4) is solved by reducing it to an equivalent finite linear system. To this end, let $\left\{\varphi_{1}, \ldots, \varphi_{N}\right\}$ be a basis of $S_{h}$, with $N=N_{h}$ the dimension of $S_{h}$. Assume

$$
x_{h}(s)=\sum_{j=1}^{N} \alpha_{j} \varphi_{j}(s), \quad s \in D .
$$

Then (1.4) is equivalent to solving for $\left\{\alpha_{j}\right\}$ in the linear system

$$
\sum_{j=1}^{N} \alpha_{j}\left\{\left(\varphi_{j}, \varphi_{i}\right)-\left(\mathscr{K} \varphi_{j}, \varphi_{i}\right)\right\}=\left(y, \varphi_{i}\right), \quad i=1, \ldots, N
$$

We will assume that this is easily solvable, although in practice the size of $N$ may necessitate an iterative method of solution. The conditioning of this linear system is examined in [5, p. 79] and [32]. The iterated Galerkin solution $x_{h}^{*}$ is obtained by substituting (2.1) into the definition (1.5).

To solve (2.2) and (1.5) in practice, usually we must numerically evaluate the integrals that occur in these formulas. Thus, introduce the numerical integration scheme

$$
\int_{D} f(t) d \sigma(t) \doteq \sum_{k=1}^{R} w_{k R} f\left(t_{k R}\right),
$$

with all $t_{k R} \in D$ and all $w_{k R} \neq 0$. Here $R=R_{h}$ is the number of node points; and we assume that the numerical integration scheme converges as $R \rightarrow \infty(h \rightarrow 0)$, for all $f \in C(D)$. Ordinarily, the weights and nodes will be written simply as $w_{k}$ and $t_{k}$, with the dependence on $R$ (or $h$ ) understood implicitly. In all cases when using (2.3) in this paper, we will assume that

$$
\text { [H1] } \quad R_{h} \geqslant N_{h}, h>0 .
$$

This will be needed for reasons that will become apparent later.

Use (2.3) to approximate all of the integrals in (2.2), applying it twice to evaluate the iterated integrals of $\left(\mathscr{K} \varphi_{j}, \varphi_{i}\right)$. Let

$$
z_{h}(s)=\sum_{j=1}^{N} \beta_{j} \varphi_{j}(s), \quad s \in D
$$

with $\beta_{1}, \ldots, \beta_{N}$ determined from the linear system

$$
\begin{aligned}
\sum_{j=1}^{N} \beta_{j}\left\{\sum_{k=1}^{R} w_{k} \varphi_{j}\left(t_{k}\right) \varphi_{i}\left(t_{k}\right)-\sum_{k=1}^{R} \sum_{l=1}^{R} w_{k} w_{l} K\left(t_{k}, t_{l}\right) \varphi_{j}\left(t_{l}\right) \varphi_{i}\left(t_{k}\right)\right\} & \\
& =\sum_{k=1}^{R} w_{k} y\left(t_{k}\right) \varphi_{i}\left(t_{k}\right), \quad i=1, \ldots, N .
\end{aligned}
$$

Note that the coefficients $\left(\varphi_{i}, \varphi_{j}\right)$ are also being approximated, as this is necessary for some of our later applications. In the earlier works [12], [30], these coefficients were assumed to be evaluated exactly; and in some cases, our work will imply the earlier results. To complete our approximation of the Galerkin method, define the 
discrete iterated Galerkin solution by

$$
z_{h}^{*}(s)=y(s)+\sum_{k=1}^{R} w_{k} K\left(s, t_{k}\right) z_{h}\left(t_{k}\right), \quad s \in D .
$$

To simplify the analysis of $z_{h}$ and $z_{h}^{*}$, introduce the following matrix notation. Let $\Phi$ be the matrix of order $N \times R$ with

$$
\Phi_{i k}=\varphi_{i}\left(t_{k}\right), \quad i=1, \ldots, N, k=1, \ldots, R .
$$

Also, define

$$
\begin{gathered}
\tilde{K}=\left[K\left(t_{k}, t_{l}\right)\right], \quad k, l=1, \ldots, R, \\
W=\operatorname{diagonal}\left[w_{1}, \ldots, w_{R}\right], \\
\mathbf{y}=\left[y\left(t_{1}\right), \ldots, y\left(t_{R}\right)\right]^{T}, \quad \boldsymbol{\beta}=\left[\beta_{1}, \ldots, \beta_{N}\right]^{T}, \\
\mathbf{z}_{h}=\left[z_{h}\left(t_{1}\right), \ldots, z_{h}\left(t_{R}\right)\right]^{T}, \quad \mathbf{z}_{h}^{*}=\left[z_{h}^{*}\left(t_{1}\right), \ldots, z_{h}^{*}\left(t_{R}\right)\right]^{T} .
\end{gathered}
$$

The linear system (2.6) can be written as

$$
\Phi W \Phi^{T} \beta-\Phi W \tilde{K} W \Phi^{T} \beta=\Phi W \mathbf{y}
$$

and

$$
\mathbf{z}_{h}=\Phi^{T} \beta
$$

The iterated solution $z_{h}^{*}$ satisfies

$$
\mathbf{z}_{h}^{*}=\mathbf{y}+\tilde{K} W \mathbf{z}_{h}
$$

With this, we have

LEMMA 2.1. $\Phi W \mathbf{z}_{h}=\Phi W \mathbf{z}_{h}^{*}$.

Proof. Multiply (2.13) by $\Phi W$, yielding

$$
\Phi W \mathbf{z}_{h}^{*}=\Phi W \mathbf{y}+\Phi W \tilde{K} W \mathbf{z}_{h} .
$$

Combining (2.12) and (2.11),

$$
\Phi W \mathbf{z}_{h}=\Phi W \mathbf{y}+\Phi W \tilde{K} W \mathbf{z}_{h} .
$$

This shows the desired result.

For the remainder of the paper, we will assume

$$
\text { [H2] } \operatorname{Rank}(\Phi)=N, \quad h>0 \text {. }
$$

The lemma leads directly to our first important result.

THEOREM 2.2. Let $N=R$, and suppose that the system (2.11) has a solution $z_{h}$. Then the iterate $z_{h}^{*}$ is a solution to the Nyström method for solving (1.1), with (2.3) as the numerical integration method:

$$
z_{h}^{*}(s)-\sum_{k=1}^{R} w_{k} K\left(s, t_{k}\right) z_{h}^{*}\left(t_{k}\right)=y(s), \quad s \in D .
$$

Proof. From the assumption $N=R, \Phi$ is a nonsingular square matrix. Then from Lemma 2.1, $\mathbf{z}_{h}=\mathbf{z}_{h}^{*}$. Combine this with the definition (2.7) to obtain (2.15), as desired. 
This theorem has consequences that will be explored in the following section. We first say something further about the matrix $\Phi$. For $R \geqslant N$, the matrix

$$
G_{h}=\Phi W \Phi^{T}
$$

is the discretization of the Gram matrix of the original Galerkin system (2.2),

$$
\Gamma_{h}=\left[\left(\varphi_{i}, \varphi_{j}\right)\right] \text {. }
$$

The discrete Gram matrix $G_{h}$ is not necessarily an approximation of $\Gamma_{h}$, as will be shown in the next section with an example, where $S_{h}$ is a space of periodic piecewise-linear functions. This means that Eq. (2.6) need not be an approximation to (2.2) in order to assure convergence of $z_{h}$ (or $z_{h}^{*}$ ) to $x$. The theory developed in Sections 4 and 5 to explain this convergence differs from the customary approach via perturbation theory, as given in [12], [30]. In certain cases $G_{h}=\Gamma_{h}$, and then our discrete Galerkin method (2.6) becomes the same as that analyzed earlier. Such cases are considered in Sections 3 and 7.

To aid in further understanding the meaning of the matrix $\Phi$, we give the following results.

Lemma 2.3. Let $N=R$. Then [H2] is true if and only if for every set of data $\left\{b_{1}, \ldots, b_{N}\right\}$, there exists a unique element $\varphi \in S_{h}$ with

$$
\varphi\left(t_{i}\right)=b_{i}, \quad i=1, \ldots, N .
$$

In fact, we have that the mapping $f \in C(D) \rightarrow \varphi \in S_{h}$, with $\varphi(s)$ interpolating $f(s)$ at the nodes $\left\{t_{j}\right\}$, is a bounded linear projection operator on $C(D)$.

Proof. This is straightforward and well known. Also, this interpolation property is well known to be true for many pairs $S_{h},\left\{t_{j}\right\}$, and thus [H2] is easily checked by considering the equivalent interpolation problem (2.18).

Remark. Let $N=R$. Then

$$
\Phi W \Phi^{T}=I \quad \text { if and only if } \Phi^{T} \Phi W=I .
$$

Proof. Again, the proof is straightforward and we omit it.

3. Applications of the Discrete Galerkin-Nyström Method. In this section we consider only the case $N_{h}=R_{h}, h>0$, with the resultant Theorem 2.2. When the discrete Galerkin solution $z_{h}$ exists, the iterated discrete Galerkin solution $z_{h}^{*}$ is simply a solution of the Nyström method for solving (1.1), with (2.3) as the integration method. Therefore, the well-developed convergence theory for the Nyström method can be used to show the convergence of the discrete Galerkin method, even in the heretofore disregarded event of the Gram matrix $\Gamma_{h}$ not being computed exactly.

The Nyström approximation method is

$$
\zeta_{h}(s)-\sum_{l=1}^{N} w_{l} K\left(s, t_{l}\right) \zeta_{h}\left(t_{l}\right)=y(s), \quad s \in D .
$$

With the assumptions that (i) Eq. (1.1) is uniquely solvable on $C(D)$, (ii) $K(s, t)$ is continuous for $s, t \in D$, and (iii) the numerical integration method (2.3) is convergent on $C(D)$, it is well known that (3.1) is uniquely solvable in $C(D)$ for all sufficiently small $h$, say $h \leqslant h_{0}$. For a development of this theory, see [5, p. 88]. 
From the error analysis of (3.1),

$$
x-\zeta_{h}=\left(I-\mathscr{K}_{h}\right)^{-1}\left(\mathscr{K} x-\mathscr{K}_{h} x\right), \quad h \leqslant h_{0},
$$

where $\mathscr{K}_{h}$ denotes the numerical integral operator in (3.1). Because of Theorem 2.2, we will henceforth identify $\zeta_{h}$ with $z_{h}^{*}$.

The Nyström method also gives a justification of the discrete Galerkin method when $N=R$. To show that the system in (2.11) is nonsingular, write it as

$$
\Phi W \Phi^{T}-\Phi W \tilde{K} W \Phi^{T}=\Phi W[I-\tilde{K} W] \Phi^{T} .
$$

The matrix $I-\tilde{K} W$ arises when solving (3.1), and it is known that

$$
\left\|(I-\tilde{K} W)^{-1}\right\| \leqslant\left\|\left(I-\mathscr{K}_{h}\right)^{-1}\right\|, \quad h \leqslant h_{0} .
$$

The matrix norm on the left side is the matrix row norm; see [5, p. 105]. Since $\Phi, W$, and $\Phi^{T}$ are also nonsingular, this shows that the system (3.1) is nonsingular; and the condition number of the matrix on the left side of (3.3) can be found from information on $\Phi, G_{h}$, and $I-\tilde{K} W$.

The result (3.2) says that the choice of $S_{h}$ is not important in the rate of convergence of $z_{h}^{*}$ to $x$, provided that [H2] is satisfied, along with $N=R$. Also, the Gram matrix $\Gamma_{h}$ does not have to be evaluated exactly in order to obtain convergence.

Application 1. Let $n>0, D=[a, b], h=(b-a) / n, s_{j}=a+j h$ for $0 \leqslant j \leqslant n$. Let $S_{h}=\mathscr{P}_{r-1}$, the set of functions that are polynomials of degree $<r$ on each subinterval $\left[s_{j-1}, s_{j}\right], j=1, \ldots, n$. The functions in $\mathscr{P}_{r-1}$ need not be continuous at the node points $s_{j}$. The dimension of $S_{h}$ is

$$
N_{h}=r n \text {. }
$$

On each subinterval $\left[s_{j-1}, s_{j}\right]$, let an integration scheme be given:

$$
\int_{s_{j-1}}^{s_{j}} f(t) d t \doteq \sum_{k=1}^{r} \nu_{k j} f\left(\tau_{k j}\right),
$$

with $s_{j-1} \leqslant \tau_{1 j}<\cdots<\tau_{r j} \leqslant s_{j}$. Assume that (3.6) has degree of precision $d$ on each subinterval $\left[s_{j-1}, s_{j}\right]$. The formula (3.6) leads naturally to an integration formula over all of $[a, b]$, and the number of integration nodes will be $R=r n$. (Note: Because of the discontinuous nature of the functions in $S_{h}$, we can allow $\tau_{1 j}=s_{j-1}$ and $\tau_{r j}=s_{j}$, while still considering $\tau_{r j}$ and $\tau_{1, j+1}$ as distinct nodes.)

The space $S_{h}$ is not contained in $C[a, b]$, so the analysis will be extended to allow functions which can be considered as continuous on each subinterval $\left[s_{j-1}, s_{j}\right]$. This can be done in several ways, as is pointed out in [9]. We will use a formulation using $L^{\infty}[a, b]$, with the point functional evaluations defined as in Section 2 of the cited paper. With this, we have the following theorem.

THEOREM 3.1. Assume (1.1) is uniquely solvable on $C[a, b]$. Further assume that $x \in C^{d+1}[a, b]$ and that $K(s, t)$ is $d+1$ times differentiable with respect to $t$. Then the iterated discrete Galerkin solution $z_{h}^{*}$ satisfies

$$
\left\|x-z_{h}^{*}\right\|_{\infty}=O\left(h^{d+1}\right) .
$$

If $d \geqslant 2 r-2$, then $\Gamma_{h}=G_{h}$. For a maximal order of convergence, choose (3.6) to be Gauss-Legendre quadrature on $\left[s_{j-1}, s_{j}\right], j=1, \ldots, n$. Then $d=2 r-1$ and

$$
\left\|x-z_{h}^{*}\right\|_{\infty}=O\left(h^{2 r}\right) \text {. }
$$


Proof. These results are a straightforward use of (3.2), together with easily derived error bounds for the numerical integration in (3.6). The fact that $G_{h}=\Gamma_{h}$ when $d \geqslant 2 r-2$ follows from the fact that the integrand in

$$
\left(\varphi_{l}, \varphi_{k}\right)=\sum_{j=1}^{n} \int_{s_{j-1}}^{s_{j}} \varphi_{l}(t) \varphi_{k}(t) d t
$$

is a polynomial of degree $\leqslant 2 r-2$ on each subinterval. The result (3.8) is the same as that given in [12, Theorem 3.6].

Application 2. Let $D$ be a piecewise-smooth surface in $\mathbf{R}^{3}$, with

$$
D=D_{1} \cup \cdots \cup D_{J} \text {. }
$$

For each $D_{j}$, assume the existence of a smooth mapping

$$
F_{j}: \hat{D}_{j} \rightarrow D_{j}, \quad j=1, \ldots, J
$$

where $\hat{D}_{j}$ is a polygonal region in the plane. For each such region $\hat{D}_{j}$, let $\left\{\hat{\Delta}_{k, j}\right\}$ be a triangulation of $\hat{D}_{j}$, and let $F_{j}\left(\hat{\Delta}_{k, j}\right)=\Delta_{k, j}$ define a corresponding triangulation of $D_{j}$. Collecting together all these triangulations of $D_{1}, \ldots, D_{J}$ one has a triangulation $\left\{\Delta_{1}, \ldots, \Delta_{n}\right\}$ of the surface $D$.

To have a standard means of defining approximations and numerical integration on $\left\{\Delta_{k}\right\}$, we introduce an alternative way of defining $\Delta_{k}$. For each $\hat{\Delta}_{k}$, let $\hat{v}_{1, k}, \hat{v}_{2, k}$, and $\hat{v}_{3, k}$ be its three vertices. Also let $\hat{e}$ be the unit simplex in the plane:

$$
\hat{e}=\{(\hat{s}, \hat{t}) \mid \hat{s}, \hat{t}, 1-\hat{s}-\hat{t} \geqslant 0\} \text {. }
$$

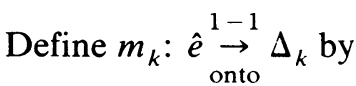

$$
m_{k}(\hat{s}, \hat{t})=F_{j}\left(\hat{u} \hat{v}_{1, k}+\hat{t} \hat{v}_{2, k}+\hat{s}_{3, k}\right), \quad(\hat{s}, \hat{t}) \in \hat{e}, \hat{u}=1-\hat{s}-\hat{t},
$$

where $\hat{\Delta}_{k} \subset \hat{D}_{j}$. We define $S_{h}$ as the set of functions that are images under $m_{k}$ of polynomials of degree $\leqslant r$ on $\hat{e}$, for $k=1, \ldots, n$. Again the space $S_{h}$ will not be contained in the continuous functions, and the analysis must be extended to $L^{\infty}(D)$ in order to carry out an error analysis.

For numerical integration, let

$$
\int_{\hat{e}} f(\hat{s}, \hat{t}) d \sigma \doteq \sum_{j=1}^{\mathscr{J}} \hat{w}_{j} f\left(\hat{s}_{j}, \hat{t}_{j}\right) .
$$

Then for integrals over $\Delta_{k}$, use

$$
\begin{aligned}
\int_{\Delta_{k}} f(Q) d S(Q) & =\int_{\hat{e}} f\left(m_{k}(\hat{s}, \hat{t})\right)\left|D_{s} m_{k} \times D_{t} m_{k}\right| d \sigma \\
& \doteq \sum_{j=1}^{\mathscr{J}} w_{k j} f\left(m_{k}\left(\hat{s}_{j}, \hat{t}_{j}\right)\right)
\end{aligned}
$$

with $w_{k j}=\hat{w}_{j}\left|D_{s} m_{k} \times D_{t} m_{k}\left(\hat{s}_{j}, \hat{t}_{j}\right)\right| . D_{s}$ denotes the partial derivative with respect to $s$, and similarly for $D_{t}$. For more information on both the triangulation of $D$ and the numerical integration (3.14), see [7], [8].

The dimension of $S_{h}$ is

$$
N=n(r+1)(r+2) / 2
$$

and the total number of integration nodes is

$$
R=n \mathscr{J} \text {. }
$$


Thus to have $N=R$, we must choose

$$
\mathscr{J}=\frac{(r+1)(r+2)}{2} \text {. }
$$

Subject to this, we choose (3.13) to maximize the degree of precision $d$ : For formulas chosen from [24], [31], we have the possibilities shown in Table 1.

Assuming (i) an integration rule of degree of precision $d$, (ii) sufficient smoothness of the functions $K(s, t)$ and $x(t)$ on each $D_{j}$, and (iii) sufficient smoothness of each $D_{j}$ (by means of the mappings $F_{j}$ ), we obtain from (3.2) that

$$
\left\|x-z_{h}^{*}\right\|_{\infty}=O\left(h^{d+1}\right)
$$

where $h$ denotes the maximum of the diameters of the triangles $\hat{\Delta}_{j}$ making up the triangulation of $\hat{D}_{1}, \ldots, \hat{D}_{J}$. This is a relatively straightforward argument, and we refer the reader to [7].

TABLE 1

Maximal degree integration rules

\begin{tabular}{l|lllr}
\hline$r$ & 0 & 1 & 2 & 3 \\
$\mathscr{J}$ & 1 & 3 & 6 & 10 \\
$d$ & 1 & 2 & 4 & 6 \\
\hline
\end{tabular}

We note that, in general, $G_{h} \neq \Gamma_{h}$, because of the presence of the Jacobian $\left|D_{s} m_{k} \times D_{t} m_{k}\right|$ in the integrand of (3.14). Nonetheless, what is referred to as superconvergence is still attained. With approximations in $S_{h}$ of degree $\leqslant r$, one would ordinarily have

$$
\left\|x-x_{h}\right\|_{\infty}=O\left(h^{r+1}\right)
$$

for the Galerkin approximation and

$$
\left\|x-x_{h}^{*}\right\|_{\infty}=O\left(h^{2 r+2}\right)
$$

for the iterated Galerkin approximation. According to Table 1 and (3.18), we do not quite attain this order of superconvergence with $z_{h}^{*}$, although there is an improvement over (3.19). More accurate numerical integration will be needed to replicate (3.20), and this will be returned to in Section 7.

Application 3. We consider two different approximating families $S_{h}$ while using the same integration rule, thus arriving at the same iterated discrete Galerkin method. Let $D$ be a smooth simple closed curve in the plane. Then $C(D)$ can be replaced by $C_{p}[0,2 \pi]$, the space of continuous periodic functions on $[0,2 \pi]$. As our first choice of $S_{h}$, use $S_{h}=\mathscr{T}_{n}$, the set of trigonometric polynomials of degree $\leqslant n$,

$$
\varphi(s)=a_{0}+\sum_{j=1}^{n}\left[a_{j} \cos (j s)+b_{j} \sin (j s)\right] .
$$

Then the dimension of $S_{h}$ is $N=2 n+1$. For the second choice of $S_{h}$, let $S_{h}=\mathscr{L}_{h}$, the set of continuous piecewise-linear periodic functions on $[0,2 \pi]$, with each $\varphi \in \mathscr{L}_{h}$ linear on $\left[t_{j-1}, t_{j}\right], j=1, \ldots, 2 n+1$,

$$
\begin{gathered}
t_{j}=j h, \quad 0 \leqslant j \leqslant 2 n, \quad h=2 \pi /(2 n+1), \\
t_{2 n+1}=t_{0}, \quad t_{2 n+2}=t_{1} .
\end{gathered}
$$

The dimension of $\mathscr{L}_{h}$ is also $N=2 n+1$. 
For the integration rule, use the trapezoidal rule with the nodes in (3.21). Then the number of distinct nodes is only $R=2 n+1$ because of the periodicity of the functions being integrated. This is a natural integration rule to use when $S_{h}=\mathscr{T}_{n}$, because the integration rule then has degree of precision $d=2 n$ and $G_{h}=\Gamma_{h}$. This would seem to preserve the rapid convergence of the Galerkin method with $S_{h}=\mathscr{T}_{h}$. For the space $S_{h}=\mathscr{L}_{h}$, however, we will not have $G_{h}=\Gamma_{h}$. For a basis of $\mathscr{L}_{h}$, use the standard 'hat' functions:

$$
\varphi_{j}(s)= \begin{cases}\left(s-t_{j-1}\right) / h, & t_{j-1} \leqslant s \leqslant t_{j} \\ \left(t_{j+1}-s\right) / h, & t_{j} \leqslant s \leqslant t_{j+1}, j=1, \ldots, 2 n+1, \\ 0, & \text { otherwise. }\end{cases}
$$

Then $\Gamma_{h}$ is almost tridiagonal (it is circulant); and $\Phi=I, G_{h}=h I . G_{h}$ is not a good approximation of $\Gamma_{h}$.

According to the standard theory of Galerkin's method, one would expect

$$
\left\|x-x_{h}\right\|_{\infty}=O\left(h^{2}\right), \quad\left\|x-x_{h}^{*}\right\|_{\infty}=O\left(h^{4}\right)
$$

with $S_{h}=\mathscr{L}_{h}$. But according to Theorem 2.2 and formula (3.2), we need only consider the integration error in examining the error in $z_{h}^{*}$, and it will coincide with the result using the more sophisticated approximation from $\mathscr{T}_{n}$. It is well known that for sufficiently smooth periodic integrands, the trapezoidal rule converges very rapidly; see [6, p. 253] and [16, p. 314]. Thus $\left\|x-z_{h}^{*}\right\|_{\infty}$ will converge very rapidly, regardless of which underlying space $S_{h}$ is being used. When going back to $z_{h}$, however, this greater speed will be lost for approximants from $\mathscr{L}_{h}$, except at the node points (since $\left.z_{h}\left(t_{i}\right)=z_{h}^{*}\left(t_{i}\right)\right)$.

4. The Discrete Orthogonal Projection. We will introduce a discrete analogue to the orthogonal projection $P_{h}$ of $L^{2}(D)$ onto $S_{h}$. Using this discrete projection, we will give an error analysis in Section 5 for the discrete Galerkin method when $R \geqslant N$. In this section the discrete projection is defined and its properties are examined. Examples with important subspaces $S_{h}$ will be given in Sections 6 and 7.

Using the numerical integration method (2.3), define

$$
\begin{gathered}
(f, g)_{h}=\sum_{k=1}^{R} w_{k} f\left(t_{k}\right) g\left(t_{k}\right), \quad f, g \in C(D), \\
\|f\|_{h}=\sqrt{(f, f)_{h}}, \quad f \in C(D) .
\end{gathered}
$$

The latter is only a seminorm on $C(D)$, but we will henceforth assume it is a norm on $S_{h}$ :

[H3] $f \in S_{h}$ and $\|f\|_{h}=0$ implies $f=0$.

We also assume

$$
\text { [H4] } \quad w_{j}>0, \quad j=1, \ldots, R \text {. }
$$

With [H4], it is usually straightforward to prove [H3]. In particular, we have the following easily proven result.

LEMMA 4.1. Let [H4] hold, and, in addition, assume that functions from $S_{h}$ possess the interpolating property (2.18) with respect to a subset of $N$ of the integration nodes used in (4.1). Then [H3] holds. 
With the definitions (4.1) and (4.2) and using [H4], it is easy to show that

$$
\begin{gathered}
\left|(f, g)_{h}\right| \leqslant\|f\|_{h}\|g\|_{h}, \quad f, g \in C(D), \\
\|f\|_{h} \leqslant c\|f\|_{\infty}, \quad c=\left[\sum_{1}^{R} w_{k}\right]^{1 / 2}, \quad f \in C(D) .
\end{gathered}
$$

Since the integration rule is convergent on $C(D)$, the constant $c$ can be bounded independent of $h$.

Define the projection operator $Q_{h}: C(D) \rightarrow S_{h}$ by

$$
\left(Q_{h} f, \psi\right)_{h}=(f, \psi)_{h} \quad \text { all } \psi \in S_{h} .
$$

To see that such a $Q_{h} f$ is uniquely defined, let $S_{h}=\operatorname{Span}\left\{\varphi_{1}, \ldots, \varphi_{N}\right\}$ and write

$$
Q_{h} f(s)=\varphi(s)=\sum_{j=1}^{N} \alpha_{j} \varphi_{j}(s), \quad s \in D .
$$

To satisfy (4.7), it is necessary and sufficient that $\alpha_{1}, \ldots, \alpha_{N}$ satisfy the linear system

$$
\sum_{j=1}^{N} \alpha_{j}\left(\varphi_{j}, \varphi_{i}\right)_{h}=\left(f, \varphi_{i}\right)_{h}, \quad i=1, \ldots, N
$$

The coefficient matrix is a Gram matrix relative to the inner product (4.1), and it is also the matrix $G_{h}$ of (2.16). To show $G_{h}$ is nonsingular, we use the standard arguments to show it is positive definite, with the aid of [H3]. Thus (4.9) is uniquely solvable and $Q_{h} f$ is uniquely defined. The linearity of (4.9), relative to $f$, will also show $Q_{h}$ is linear; and the uniqueness of $Q_{h} f$ shows $Q_{h}$ is a projection, i.e., $Q_{h}^{2}=Q_{h}$.

As a first indication of the usefulness of $Q_{h}$, we give the following result.

Lemma 4.2. Let $z_{h}$ and $z_{h}^{*}$ be the discrete Galerkin and discrete iterated Galerkin solutions, from (2.5)-(2.7), assuming they exist. Then

$$
z_{h}=Q_{h} z_{h}^{*},
$$

in analogy with (1.11) for the original Galerkin method.

Proof. Recall Lemma 2.1, that $\Phi W \mathbf{z}_{h}=\Phi W \mathbf{z}_{h}^{*}$.

Equating corresponding elements,

$$
\begin{aligned}
\sum_{k=1}^{R} w_{k} \varphi_{i}\left(t_{k}\right) z_{h}\left(t_{k}\right) & =\sum_{k=1}^{R} w_{k} \varphi_{i}\left(t_{k}\right) z_{h}^{*}\left(t_{k}\right), \quad i=1, \ldots, N, \\
\left(z_{h}, \varphi_{i}\right)_{h} & =\left(z_{h}^{*}, \varphi_{i}\right)_{h}, \quad i=1, \ldots, N .
\end{aligned}
$$

Since $\left\{\varphi_{1}, \ldots, \varphi_{N}\right\}$ is a basis of $S_{h}$, this says

$$
\left(z_{h}, \psi\right)_{h}=\left(z_{h}^{*}, \psi\right)_{h} \quad \text { all } \psi \in S_{h} \text {. }
$$

From $z_{h} \in S_{h}$, the uniqueness of $Q_{h} z_{h}^{*}$ combined with this latter result proves (4.10).

Some additional properties of $Q_{h}$ are given in the following

Lemma 4.3. (a) $Q_{h}$ is selfadjoint on $C(D)$, relative to the discrete inner product (4.1):

$$
\left(Q_{h} f, g\right)_{h}=\left(f, Q_{h} g\right)_{h}, \quad f, g \in C(D) \text {. }
$$


(b)

$$
\left\|Q_{h} f\right\|_{h} \leqslant\|f\|_{h}, \quad f \in C(D) .
$$

(c) If the family $\left\{Q_{h}: h>0\right\}$ is uniformly bounded on $C(D)$, using the usual operator norm induced by the uniform norm $\|\cdot\|_{\infty}$, then

$$
\left\|f-Q_{h} f\right\|_{\infty} \leqslant c \cdot \underset{\varphi \in S_{h}}{\operatorname{minimum}}\|f-\varphi\|_{\infty},
$$

with $c$ independent of $h$ and $f$.

(d) When $N=R$, the projection $Q_{h}$ is the interpolating projection from $C(D)$ onto $S_{h}$, i.e.,

$$
\left(Q_{h} f\right)\left(t_{i}\right)=f\left(t_{i}\right), \quad i=1, \ldots, N .
$$

Proof. (a) From (4.7) with $\psi=Q_{h}$ g,

$$
\left(Q_{h} f, Q_{h} g\right)_{h}=\left(f, Q_{h} g\right)_{h} \text {. }
$$

Similarly,

$$
\left(Q_{h} g, Q_{h} f\right)_{h}=\left(g, Q_{h} f\right)_{h} .
$$

Using the symmetry of the inner product, result (4.11) is proven.

(b)

$$
\begin{aligned}
\left\|Q_{h} f\right\|_{h}^{2} & =\left(Q_{h} f, Q_{h} f\right)_{h} \\
& =\left(f, Q_{h} f\right)_{h}, \quad \text { using } \psi=Q_{h} f \text { in (4.7) } \\
& \leqslant\|f\|_{h}\left\|Q_{h} f\right\|_{h}, \quad \text { using }(4.5) .
\end{aligned}
$$

If $Q_{h} f \neq 0$, cancel $\left\|Q_{h} f\right\|_{h}$ to prove (4.12). If $Q_{h} f=0$, then (4.12) is trivially true.

(c) Let

$$
q=\underset{h>0}{\operatorname{Supremum}}\left\|Q_{h}\right\|<\infty .
$$

Then for any $\varphi \in S_{h}$,

$$
\begin{gathered}
f-Q_{h} f=f-\varphi+Q_{h} \varphi-Q_{h} f=\left(I-Q_{h}\right)(f-\varphi), \\
\left\|f-Q_{h} f\right\|_{\infty} \leqslant(1+q)\|f-\varphi\|_{\infty} .
\end{gathered}
$$

Let $c=1+q$, and form the minimum of the right side over $S_{h}$ to prove (4.13).

(d) The system (4.9), defining $Q_{h} f$, can be written as

$$
G_{h} \boldsymbol{\alpha}=\Phi W \mathbf{f}, \quad \mathbf{f}=\left[f\left(t_{1}\right), \ldots, f\left(t_{N}\right)\right]^{T} .
$$

Using (2.16) for $G_{h}$,

$$
\Phi W \Phi^{T} \boldsymbol{\alpha}=\Phi W \mathbf{f} .
$$

Since $\Phi$ and $W$ are nonsingular, we have

$$
\Phi^{T} \boldsymbol{\alpha}=\mathbf{f} .
$$

But from (4.8), with $s=t_{i}$, we have

$$
Q_{h} f\left(t_{i}\right)=\left(\Phi^{T} \mathbf{\alpha}\right)_{i}=(\mathbf{f})_{i}=f\left(t_{i}\right), \quad i=1, \ldots, N,
$$

and thus the result is proven.

Part (c) shows that $Q_{h} f \rightarrow f$ in $C(D)$ if (i) the family $\left\{Q_{h}: h>0\right\}$ is uniformly bounded on $C(D)$, and (ii) the family $\left\{S_{h}: h>0\right\}$ approximates all elements of $C(D)$. Conversely, if $Q_{h} f \rightarrow f$ for all $f \in C(D)$, then $\left\{Q_{h}\right\}$ is uniformly bounded 
by the principle of uniform boundedness. We examine next the general problem of bounding the family $\left\{Q_{h}\right\}$ for some important approximating families $\left\{S_{h}\right\}$.

In general, we need to bound $Q_{h} f$ in (4.8), subject to $\alpha_{1}, \ldots, \alpha_{N}$ satisfying (4.9). The choice of the basis $\left\{\varphi_{1}, \ldots, \varphi_{N}\right\}$ of $S_{h}$ is at our disposal. Since the coefficient matrix of (4.8) is $G_{h}$, a bound for $Q_{h}$ can be produced by using

$$
\begin{aligned}
\|\boldsymbol{\alpha}\|_{\infty} & \leqslant\left\|G_{h}^{-1}\right\| \underset{1 \leqslant i \leqslant N}{\operatorname{Max}}\left|\left(f, \varphi_{i}\right)_{h}\right| \\
& \leqslant\left\|G_{h}^{-1}\right\|\|f\|_{\infty} \operatorname{Max}_{1 \leqslant i \leqslant N} \sum_{k=1}^{N} w_{k}\left|\varphi_{i}\left(t_{k}\right)\right|
\end{aligned}
$$

with $\alpha=\left[\alpha_{1}, \ldots, \alpha_{N}\right]^{T}$. The matrix norm used in $\left\|G_{h}^{-1}\right\|$ is the row norm. Combining (4.8) and (4.18),

$$
\begin{gathered}
\left\|Q_{h} f\right\|_{\infty} \leqslant c\|f\|_{\infty}, \\
c=\left\|G_{h}^{-1}\right\|\left[\operatorname{Max}_{s \in D} \sum_{i=1}^{N}\left|\varphi_{i}(s)\right|\right]\left[\operatorname{Max}_{1 \leqslant i \leqslant N} \sum_{k=1}^{R} w_{k}\left|\varphi_{i}\left(t_{k}\right)\right|\right] .
\end{gathered}
$$

Thus the problem of bounding $Q_{h}$ is reduced to that of bounding $G_{h}^{-1}$, at least in the case when the underlying subspaces $S_{h}$ are of finite element type. For then,

$$
\operatorname{Max}_{s \in D} \sum_{i=1}^{N}\left|\varphi_{i}(s)\right|
$$

can be reasonably assumed to be bounded independent of $h$; while for the last term in (4.20), an estimate

$$
\underset{1 \leqslant i \leqslant N}{\operatorname{Max}} \sum_{k=1}^{R} w_{k}\left|\varphi_{i}\left(t_{k}\right)\right| \leqslant c h^{\delta}
$$

could be established.

Bounds on $\left\|\Gamma_{h}^{-1}\right\|$ were studied in [10], [18] in case of spline spaces $S_{h}$, and in [17], [19] for finite element spaces over subregions in $\mathbf{R}^{n}$. The possibility of defining the discrete inner product and the corresponding projection was mentioned in the latter paper; and the discrete projection onto spline spaces in conjunction with collocation methods at Gaussian points was used in [21], [22]. Unlike the latter two papers and aforementioned works [12], [30], we do not require $G_{h}=\Gamma_{h}$ in order to establish convergence $\left\|x-z_{h}^{*}\right\| \rightarrow 0$.

Anticipating the superconvergence results for the discrete iterate $z_{h}^{*}$, we next show that the discrete Galerkin solution $z_{h}$ inherits certain superconvergence properties of $z_{h}^{*}$.

LeMma 4.4. Let $z_{h}$ and $z_{h}^{*}$ be defined as in Lemma 4.2. Then

$$
\left\|Q_{h} x-z_{h}\right\|_{h} \leqslant c\left\|x-z_{h}^{*}\right\|_{\infty}
$$

where $c$ is the constant in (4.6).

Proof. Using consecutively (4.10), (4.12), and (4.6), one has

$$
\left\|Q_{h} x-z_{h}\right\|_{h}=\left\|Q_{h}\left(x-z_{h}^{*}\right)\right\|_{h} \leqslant\left\|x-z_{h}^{*}\right\|_{h} \leqslant c\left\|x-z_{h}^{*}\right\|_{\infty} .
$$

Assuming (4.19), we state a stronger result. 
Lemma 4.5. Assume (4.19) holds, i.e., $\left\|Q_{h} f\right\|_{\infty} \leqslant c\|f\|_{\infty}$ for all $f \in C(D)$ with $c$ independent of $h$. Then

$$
\left\|Q_{h} x-z_{h}\right\|_{\infty} \leqslant c\left\|x-z_{h}^{*}\right\|_{\infty} .
$$

Proof. In (4.19), set $f=x-z_{h}^{*}$; and then use

$$
\left\|Q_{h} x-z_{h}\right\|_{\infty}=\left\|Q_{h}\left(x-z_{h}^{*}\right)\right\|_{\infty} \leqslant c\left\|x-z_{h}^{*}\right\|_{\infty} .
$$

With $N=R, Q_{h}$ is just the interpolating projection. Thus (4.22) extends the result of Richter [26], on the superconvergence of $\left(x-x_{h}\right)\left(t_{i}\right)$ for collocation at Gaussian points, to the case of the discrete Galerkin method.

Thus we see that the convergence of the discrete Galerkin method hinges on availability of the bound (4.19). This will be discussed in Sections 6 and 7. But first we introduce the formalism for handling the fully discretized Galerkin method.

5. General Error Analysis. Recall the numerical integral operator $\mathscr{K}_{h}$ based on the integration rule (2.3),

$$
\left(\mathscr{K}_{h} f\right)(s)=\sum_{j=1}^{R} w_{j} K\left(s, t_{j}\right) f\left(t_{j}\right), \quad s \in D .
$$

Using the notion of the discrete inner product (4.1), we also have

$$
\left(\mathscr{K}_{h} f\right)(s)=\left(k_{s}, f\right)_{h},
$$

where $k_{s}=K(s, \cdot)$.

LEMMA 5.1. The discrete Galerkin method (2.5)-(2.6) can be written equivalently as

$$
\left(I-Q_{h} \mathscr{K}_{h}\right) z_{h}=Q_{h} y, \quad z_{h} \in C(D) .
$$

Moreover, the discrete iterated Galerkin solution satisfies

$$
\left(I-\mathscr{K}_{h} Q_{h}\right) z_{h}^{*}=y .
$$

Proof. From (5.3), $z_{h}=Q_{h}\left(y+\mathscr{K}_{h} z_{h}\right) \in S_{h}$. Using this, rewrite (5.3) as

$$
Q_{h}\left[\left(I-\mathscr{K}_{h}\right) z_{h}-y\right]=0, \quad z_{h} \in S_{h} .
$$

This is equivalent to saying that $z_{h} \in S_{h}$ and that it satisfies

$$
\left(\left(I-\mathscr{K}_{h}\right) z_{h}-y, \psi\right)_{h}=0 \quad \text { all } \psi \in S_{h} .
$$

Choose a basis $\left\{\varphi_{1}, \ldots, \varphi_{N}\right\}$ of $S_{h}$, and successively set $\psi=\varphi_{i}, i=1, \ldots, N$. This leads directly to the formulation (2.5)-(2.6), showing the equivalence with the formula (5.3).

As to $z_{h}^{*}$, the definition (2.7) is equivalent to

$$
z_{h}^{*}=y+\mathscr{K}_{h} z_{h},
$$

from which $Q_{h} z_{h}^{*}=Q_{h} y+Q_{h} \mathscr{K}_{h} z_{h}=z_{h}$, supplying another proof of Lemma 4.2. Now replacing $z_{h}$ with $Q_{h} z_{h}^{*}$ in (5.5), we prove (5.4).

Solvability of the two equations (5.3) and (5.4) follows easily from the standard theory of collectively compact families of operators, [1], [5].

ThEOREM 5.2. Assume the integral equation $(I-\mathscr{K}) x=y$ is uniquely solvable for every $y \in C(D)$, and assume $K(s, t)$ is continuous for $s, t \in D$. Let

$$
\left\{S_{h} \subset C(D): h>0\right\}
$$


be a sequence of approximating subspaces with the property that for each $f \in C(D)$,

$$
\rho_{h}(f)=\min _{\varphi \in S_{h}}\|f-\varphi\|_{\infty} \rightarrow 0 \text { as } h \rightarrow 0 .
$$

Finally, assume that the discrete projections $\left\{Q_{h}: h>0\right\}$ are uniformly bounded,

$$
q \equiv \sup _{h>0}\left\|Q_{h}\right\|<\infty .
$$

Then $\left\{Q_{h} \mathscr{K}_{h}\right\}$ and $\left\{\mathscr{K}_{h} Q_{h}\right\}$ each form a pointwise convergent and collectively compact family of operators. Hence, for all sufficiently small $h$, the operators $I-Q_{h} \mathscr{K}_{h}$ and $I-\mathscr{K}_{h} Q_{h}$ are invertible on $C(D)\left(\right.$ for, say, $\left.h \leqslant h_{0}\right)$, and

$$
\left\|\left(I-Q_{h} \mathscr{K}_{h}\right)^{-1}\right\| \leqslant B, \quad\left\|\left(I-\mathscr{K}_{h} Q_{h}\right)^{-1}\right\| \leqslant B<\infty .
$$

Moreover,

$$
\left(I-Q_{h} \mathscr{K}_{h}\right)\left(x-z_{h}\right)=\left(I-Q_{h}\right)\left(y+\mathscr{K}_{h} x\right)+\left(\mathscr{K}-\mathscr{K}_{h}\right) x
$$

and

$$
\left(I-\mathscr{K}_{h} Q_{h}\right)\left(x-z_{h}^{*}\right)=\mathscr{K} x-\mathscr{K}_{h} Q_{h} x .
$$

Thus both $\left\|x-z_{h}\right\|_{\infty}$ and $\left\|x-z_{h}^{*}\right\|$ tend to 0 as $h \rightarrow 0$.

Proof. We concentrate on the equation (5.4). Equation (5.3) can be dealt with in a similar manner. We show that $\left\{\mathscr{K}_{h} Q_{h}: h>0\right\}$ is a pointwise convergent and collectively compact family of operators on $C(D)$. Then the general theory of collectively compact operators can be used to complete the proof (e.g., see [1] or [5, p. 96]).

From (5.7), (5.8), and (4.17),

$$
\left\|f-Q_{h} f\right\|_{\infty} \leqslant(1+q) \rho_{h}(f)
$$

proving that $Q_{h} f \rightarrow f$ as $h \rightarrow 0$, for all $f \in C(D)$. Then

$$
\mathscr{K} f-\mathscr{K}_{h} Q_{h} f=\left[\mathscr{K}_{f}-\mathscr{K}_{h} f\right]+\mathscr{K}_{h}\left[f-Q_{h} f\right] .
$$

It is straightforward to show that $\left\|\mathscr{K} f-\mathscr{K}_{h} f\right\|_{\infty} \rightarrow 0$ (see [5, p. 90]); and then the principle of uniform boundedness implies $\left\{\mathscr{K}_{h}\right\}$ is uniformly bounded. Combining the latter with (5.12), the last term in (5.13) also goes to zero, completing the proof that $\mathscr{K}_{h} Q_{h} f \rightarrow \mathscr{K} f$.

The collective compactness of $\left\{\mathscr{K}_{h} Q_{h}: h>0\right\}$ will follow by standard arguments. Combined with the pointwise convergence of $\left\{\mathscr{K}_{h} Q_{h}\right\}$, the remaining results follow from known theory [1], [5].

We now give another proof of Theorem 2.2, within this new framework.

COROLlaRy 5.3. When $N=R$, the discrete iterated Galerkin solution results from a Nyström approximation of the iniegral equation (1.1).

Proof. From Lemma 4.3(d), we have $\mathscr{K}_{h} Q_{h} f(s)=\mathscr{K}_{h} f(s)$ for all $f \in C(D)$. Thus formula (5.4) for $z_{h}^{*}$ reduces to

$$
\left(I-\mathscr{K}_{h}\right) z_{h}^{*}=y
$$

the Nyström method.

To obtain superconvergence results for $z_{h}^{*}$ to $x$ in the original Galerkin method, examine the error term in (5.11). This reduces to examining the error

$$
\mathscr{K} x(s)-\mathscr{K}_{h} Q_{h} x(s) .
$$


LemMa 5.4. Let $k_{s}(t)=K(s, t), s, t \in D$. Then

$$
\mathscr{K} x(s)-\mathscr{K}_{h} Q_{h} x(s)=\left[\mathscr{K} x(s)-\mathscr{K}_{h} x(s)\right]+\left(\left(I-Q_{h}\right) k_{s},\left(I-Q_{h}\right) x\right)_{h}
$$

and

$$
\begin{aligned}
\left|\mathscr{K} x(s)-\mathscr{K}_{h} Q_{h} x(s)\right| \leqslant & \left|\mathscr{K} x(s)-\mathscr{K}_{h} x(s)\right| \\
& +\left\|\left(I-Q_{h}\right) k_{s}\right\|_{h, 1} \cdot\left\|\left(I-Q_{h}\right) x\right\|_{h, \infty} .
\end{aligned}
$$

The seminorms are

$$
\|f\|_{h, 1}=\sum_{j=1}^{R} w_{j}\left|f\left(t_{j}\right)\right|, \quad\|f\|_{h, \infty}=\underset{1 \leqslant j \leqslant R}{\operatorname{Maximum}}\left|f\left(t_{j}\right)\right| .
$$

Proof. Write

$$
\mathscr{K} x(s)-\mathscr{K}_{h} Q_{h} x(s)=\left[\mathscr{K} x(s)-\mathscr{K}_{h} x(s)\right]+\mathscr{K}_{h}\left(I-Q_{h}\right) x(s) .
$$

Then note that from (5.2),

$$
\begin{aligned}
\mathscr{K}_{h}\left(I-Q_{h}\right) x(s) & =\left(k_{s},\left(I-Q_{h}\right) x\right)_{h}=\left(k_{s},\left(I-Q_{h}\right)^{2} x\right)_{h} \\
& =\left(\left(I-Q_{h}\right) k_{s},\left(I-Q_{h}\right) x\right)_{h} .
\end{aligned}
$$

The last two steps used the facts that $I-Q_{h}$ is a projection and that it is selfadjoint from Lemma 4.3(a). (5.15) follows easily from (5.14).

This shows the quantities that must be examined in order to obtain convergence results for $z_{h}^{*}$, namely, (1) $\mathscr{K} x-\mathscr{K}_{h} x$, (2) $x-Q_{h} x$, and (3) $\left(I-Q_{h}\right) k_{s}$. The first is simply the numerical integration error. The second and third are the errors in using the projection of $C(D)$ into $S_{h}$, and these are examined for particular cases in the next two sections.

When $S_{h}$ contains only piecewise-continuous functions, the above arguments can still be used. Note that if $S_{h} \subset L^{\infty}(D)$, then $\mathscr{K}: L^{\infty}(D) \rightarrow C(D)$ implies that $\mathscr{K}_{h} Q_{h}: C(D) \rightarrow C(D)$, and $z_{h}^{*}$ will still be a continuous function. The discrete solution $z_{h}$ will not be continuous, but the following bound in (5.17) is still valid.

Note also that for the difference $x-z_{h}$ one has

$$
\left\|x-z_{h}\right\|_{\infty}=\left\|x-Q_{h} z_{h}^{*}\right\|_{\infty} \leqslant\left\|x-Q_{h} x\right\|_{\infty}+\left\|Q_{h}\right\|\left\|x-z_{h}^{*}\right\|_{\infty},
$$

which provides a way other than (5.10) to estimate $\left\|x-z_{h}\right\|_{\infty}$. As the first application of the above lemma, consider the simple case of a smooth kernel $K(s, t)$ and a smooth right-hand side $y$. Assume also that for smooth $f$,

$$
\inf _{\varphi \in S_{h}}\|f-\varphi\|_{\infty}=O\left(h^{l}\right) \text {; }
$$

and assume the integration formula used is of degree of precision $d$. Then under the assumption that $\left\{Q_{h}\right\}$ is uniformly bounded,

$$
\begin{aligned}
\left\|x-z_{h}\right\|_{\infty} & =O\left(h^{\min (l, d+1)}\right), \\
\left\|x-z_{h}^{*}\right\|_{\infty} & =O\left(h^{\min (2 l, d+1)}\right) .
\end{aligned}
$$

(5.19) actually coincides with a result by Chandler [12], while (5.18) improves the corresponding result of his in the event we are concerned with, i.e., when the same integration formula is used in defining $Q_{h}$ and $\mathscr{K}_{h}$. 
While we are on the case of smooth kernels, let us use (5.14) to make a remark that parallels one in [28]. Namely, the rate of convergence observed in (5.19) remains the same for all derivatives of $x-z_{h}^{*}$, i.e.,

$$
\left\|D^{\alpha}\left(x-z_{h}^{*}\right)\right\|_{\infty}=O\left(h^{\min (2 l, d+1)}\right),
$$

where $\alpha$ is a multi-index. This follows from (i) the fact that the uniform boundedness of $\left(I-\mathscr{K}_{h} Q_{h}\right)^{-1}$ in $C(D), h \leqslant h_{\infty}$, implies their uniform boundedness in $C^{k}(D)$, the space of $k$ times continuously differentiable functions, and (ii) the easy formula

$$
\left\|D^{\alpha} \mathscr{K}_{h}\left(I-Q_{h}\right) x\right\|_{\infty}=\max _{s}\left(\left(I-Q_{h}\right) k_{s}^{\alpha},\left(I-Q_{h}\right) x\right)_{h},
$$

where $k_{s}^{\alpha}=D_{s}^{\alpha} K(s, \cdot)$.

6. Bounds on $G_{n}^{-1}$. See the Supplements section at the end of this issue.

7. Applications. In this section, we derive the bound in (4.19)-(4.20), using the results of the preceding section. To define the projection $Q_{h}$, we have to specify the spaces $S_{h}$ and the numerical quadratures (2.3).

We consider finite element spaces as described in Section 6. Starting with an integration formula over the reference element,

$$
\int_{\hat{e}} \hat{\varphi}(\hat{t}) \hat{d} t \doteq \sum_{j=1}^{\mathscr{J}} \hat{w}_{j} \hat{\varphi}\left(\hat{t}_{j}\right),
$$

we define the composite formula over $D$,

$$
\int_{D} \varphi(t) d t \doteq \sum_{k=1}^{E} \sum_{j=1}^{\mathscr{J}} w_{j k} \varphi\left(t_{j k}\right),
$$

where $t_{j k}=F_{k}\left(\hat{t}_{j}\right)$, and $w_{j k}=\hat{w}_{j}\left|D F_{k}\left(\hat{t}_{j}\right)\right|, k=1, \ldots, E, j=1, \ldots, \mathscr{J}$, in accordance with (3.13)-(3.14).

Using (7.2), we define the scalar product $(\cdot, \cdot)_{h}$ as in (4.1) and (6.2), with

$$
(\varphi, \psi)_{e_{k}}=\sum_{j=1}^{\mathscr{J}} w_{j k} \varphi\left(t_{j k}\right) \psi\left(t_{j k}\right) .
$$

This clearly satisfies $(6.10)$ and $(6.11)$, if the scalar product $(\cdot, \cdot)^{\wedge}$ is defined by means of

$$
(\hat{\varphi}, \hat{\psi}) \hat{=} \sum_{j=1}^{\mathscr{J}} \hat{w}_{j} \hat{\varphi}\left(\hat{t}_{j}\right) \hat{\psi}\left(\hat{t}_{j}\right) .
$$

To state a general result, we have to impose two additional conditions, in addition to (a)-(j) of Section 6.

$$
F_{k} \text { is continuously differentiable and }
$$

$$
\max _{\hat{i} \in \hat{e}}\left|D F_{k}(\hat{t})\right| \leqslant \kappa m\left(e_{k}\right), \quad k=1, \ldots, E .
$$

In case $F_{k}$ is an affine mapping as in (6.11), this follows from $\left|D F_{k}\right|=m\left(e_{k}\right) / m(\hat{e})$.

$$
\text { Let } M_{j}=\left\{k: C_{j} \supset e_{k}\right\} \text {. Then }\left|M_{j}\right| \leqslant M, j=1, \ldots, N \text {. }
$$

This is easily verified for all commonly used finite element spaces.

We can now state the following general result. 
THEOREM 7.1. Let spaces $S_{h}$ be defined as in (a)-(1), where for definiteness, we set $h=\max \left(d\left(e_{k}\right)\right), k=1, \ldots, E$. Then with $(\cdot, \cdot)_{h}$ defined as above and $Q_{h}$ as in (4.7),

$$
\left\|Q_{h}\right\| \leqslant C
$$

independent of $h$.

Proof. We have to estimate the three terms in (4.20). First, using (j), we get

$$
\begin{aligned}
\underset{s \in D}{\operatorname{Max}} \sum_{i=1}^{N}\left|\varphi_{i}(s)\right| & =\underset{k=1, \ldots, E}{\operatorname{Max}} \underset{E \in e_{k}}{\operatorname{Max}} \sum_{i=1}^{N}\left|\varphi_{i}(s)\right| \\
& =\underset{k=1, \ldots, E}{\operatorname{Max}} \underset{s \in e_{k}}{\operatorname{Max}} \sum_{i \in I_{k}}\left|\varphi_{i}(s)\right| \\
& \leqslant M \cdot \underset{k=1, \ldots, E}{\operatorname{Max}} \underset{s \in e_{k}}{\operatorname{Max}} \operatorname{Max}_{i \in I_{k}}\left|\varphi_{i}(s)\right| \\
& \leqslant M \cdot \underset{j=1, \ldots, \nu}{\operatorname{Max}} \underset{\hat{s} \in \hat{e}}{\operatorname{Max}}\left|\hat{\varphi}_{j}(\hat{s})\right| \leqslant C \cdot M,
\end{aligned}
$$

for a constant $C$ independent of $h$.

For all $i=1, \ldots, N$ and $k \in M_{i}$, denote the progenitor of $\varphi_{i}$ over $e_{k}$ as $\hat{\varphi}_{i_{k}}$ :

$$
\hat{\varphi}_{i_{k}}=\left.\varphi_{i}\right|_{e_{k}} \circ F_{k}
$$

as in (6.14). Using this notation, we next estimate the last factor in (4.20). Thus, for all $i=1, \ldots, N$, we see that

$$
\begin{aligned}
\sum_{k=1}^{R} w_{k}\left|\varphi_{i}\left(t_{k}\right)\right| & =\sum_{k=1}^{E} \sum_{j=1}^{\mathscr{J}} w_{j k}\left|\varphi_{i}\left(t_{j k}\right)\right| \\
& =\sum_{k \in M_{i}} \sum_{j=1}^{\mathscr{J}} w_{j k}\left|\hat{\varphi}_{i_{k}}\left(\hat{t}_{j}\right)\right|, \quad \text { from }(\mathrm{j}) \\
& \leqslant \kappa \sum_{k \in M_{i}} \sum_{j=1}^{\mathscr{J}} \hat{w}_{j} m\left(e_{k}\right)\left|\hat{\varphi}_{i_{k}}\left(\hat{t}_{j}\right)\right|, \quad \text { from }(\mathrm{k}) \\
& \leqslant \kappa \cdot \operatorname{Max}_{l=1, \ldots, \nu} \sum_{k \in M_{i}} \sum_{j=1}^{\mathscr{J}}\left|\hat{\varphi}_{l}\left(\hat{t}_{j}\right)\right| \cdot \underset{k=1, \ldots, E}{\operatorname{Max}} m\left(e_{k}\right) \\
& \leqslant \kappa \cdot M \cdot c h^{\bar{n}}=C h^{\bar{n}},
\end{aligned}
$$

where $\bar{n}=n$ or $\bar{n}=n-1$ as in (h), and $C$ is independent of $h$.

Finally, applying Lemma 6.3, (k), and the same bound on $m\left(e_{k}\right)$ as in (7.7), we obtain through Theorem 6.1 the estimate

$$
\left\|G_{h}^{-1}\right\| \leqslant C h^{-\bar{n}}
$$

with $C$ independent of $h$.

Combining (4.20), (7.6)-(7.8), we finish the proof.

Examples. Consider the unit simplex $\hat{e}$, as in (3.11). Let $\hat{v}_{1}, \hat{v}_{2}, \hat{v}_{3}$ be its vertices while $\hat{v}_{12}, \hat{v}_{23}, \hat{v}_{13}$ are the midpoints of its sides. First we study the element $\left\{\hat{e}, \hat{\Sigma}^{0}, \mathscr{P}_{1}\right\}$, where $\mathscr{P}_{1}$ is the set of polynomials of degree less than 2 , and $\hat{\Sigma}^{0}=\left\{\delta_{\hat{v}_{1}}, \delta_{\hat{v}_{2}}, \delta_{\hat{v}_{3}}\right\}$. The scalar products are defined with

$$
(\hat{\varphi}, \hat{\psi})^{\wedge}=\frac{1}{6} \sum_{i=1}^{3} \hat{\varphi}\left(\hat{v}_{i}\right) \hat{\psi}\left(\hat{v}_{i}\right)
$$


The space $S_{h}$ consists of piecewise-linear continuous functions, and the discrete iterated Galerkin method reduces to the Nyström method with nodes at vertices of the triangulation.

If one chooses instead $\hat{\Sigma}^{0}=\left\{\delta_{\hat{v}_{12}}, \delta_{\hat{v}_{23}}, \delta_{\hat{v}_{13}}\right\}$ and replaces (7.9) with

$$
(\hat{\varphi}, \hat{\psi})^{\wedge}=\frac{1}{6} \sum_{\substack{i, j=1 \\ i \neq j}}^{3} \hat{\varphi}\left(\hat{v}_{i j}\right) \hat{\psi}\left(\hat{v}_{i j}\right),
$$

then $S_{h}$ will consist of piecewise-linear functions, continuous at the midpoints of the sides of the triangular elements. The discrete iterated Galerkin method coincides with the Nyström method with nodes at these points. Again, although (7.10) is exact for the finite elements concerned, $Q_{h}$ does not reduce to the customary orthogonal projection $P_{h}$.

It immediately follows from the general theory that, taking operators $\mathscr{K}$ with a smooth kernel, we have $\left\|x-z_{h}^{*}\right\|_{\infty} \leqslant c h^{2}$ in the first case, while $\left\|x-z_{h}^{*}\right\|_{\infty} \leqslant c h^{3}$ in the second case, since the degree of precision of the quadrature in (7.9) is 1 , while that of the quadrature in (7.10) is 2 . Note that $\left\|x-x_{h}^{*}\right\|_{\infty} \leqslant c h^{4}$, from which we see that, though the discrete iterate $z_{h}^{*}$ converges faster than $z_{h}$ (or for that matter $x_{h}$ ), it does not attain the full superconvergence of $x_{h}^{*}$. As we see, to achieve this, it does not suffice to choose an integration formula for which the integral in (7.4) is evaluated exactly. To obtain the convergence rate $O\left(h^{4}\right)$ for $z_{h}^{*}$, an integration formula with degree of precision $d \geqslant 3$ is needed. From [24], there exists a formula with $d=3$ that has 4 nodes.

As the last example of finite element spaces, we consider the piecewise-smooth surface $D$ from (3.9). The triangulation is defined by (3.10)-(3.12), while the scalar products $(\cdot, \cdot)_{e_{i}}$ are defined by means of the integration (3.13)-(3.14). The kernel of the integral operator $\mathscr{K}$ is assumed to be smooth on each of the patches $D_{j}$. The same holds for the solution $x$, provided the right-hand side $y$ is sufficiently smooth.

Theorem 7.1 applies directly; thus we have that (4.19) holds. Convergence estimates easily follow from $(5.14),(5.17)$. The difference $\left(\mathscr{K}-\mathscr{K}_{h}\right) x(s)$ is bounded as in [7], [8]; we have as before

$$
\left\|x-z_{h}\right\|_{\infty} \leqslant c\left(h^{d+1}+h^{r+1}\right) \text { and }\left\|x-z_{h}^{*}\right\|_{\infty} \leqslant c\left(h^{d+1}+h^{2(r+1)}\right) .
$$

To attain the rate of convergence of the exact Galerkin iterate $x_{h}^{*}$, which is $h^{2(r+1)}$, one has to choose an integration formula with

$$
d+1 \geqslant 2(r+1) \text {. }
$$

Finally, note again that (7.11) does not imply $Q_{h}=P_{h}$.

Lastly, we would like to remark on the applicability of the developed theory to the spline spaces. The crucial assumption (j) holds for the spline spaces $S(r, k)$ with $2 k \leqslant r-1$, where

$$
S(r, k)=\left\{f \in C^{k}[a, b]:\left.f\right|_{\left[t_{i-1}, t_{t}\right]} \in \mathscr{P}_{r}\right\} .
$$

Here, $\Delta: a=t_{0}<\cdots<t_{m}=b$ is a partition of a finite interval $[a, b], \mathscr{P}_{r}$ is the set of polynomials of degree less than $r$. When $2 k=r-1$ one has a space of Hermite splines; with $2 k<r-1$ the splines are less continuous than in the latter case. 
Thus we can formulate the following

COROllary 7.2. Assume for $S_{h}=S(r, k)$ with $2 k \leqslant r-1$ that (a)-(k) hold. Then with $(\cdot, \cdot)_{h}$ defined by (6.2) and $Q_{h}$ by $(4.7)$,

$$
\left\|Q_{h}\right\| \leqslant C
$$

independent of $h$.

Example. Let $K$ be an operator with a smooth kernel. Then using the above-defined spline spaces $S_{h}$ of Corollary 7.2, we have two estimates for the exact Galerkin and iterated Galerkin methods:

$$
\left\|x-x_{h}\right\|_{\infty} \leqslant c h^{r}
$$

and

$$
\left\|x-x_{h}^{*}\right\|_{\infty} \leqslant c h^{2 r},
$$

provided the right-hand side $y \in C^{r}[a, b]$.

If the quadrature in (7.1) has degree of precision $d$, then from (5.14) we immediately have

$$
\left\|x-z_{h}^{*}\right\|_{\infty} \leqslant c\left(h^{d+1}+h^{2 r}\right)
$$

and combined with (5.17),

$$
\left\|x-z_{h}\right\|_{\infty} \leqslant c\left(h^{d+1}+h^{r}\right) .
$$

Then using numerical integration maintains the accuracy of the Galerkin method if $d \geqslant r-1$. To preserve the accuracy of the iterate, we need $d \geqslant 2 r-1$; although with $d>r-1$, the discrete iterate will exhibit superconvergence. Compare with [12].

Remark. Consider more general spline spaces $S_{h}=S(r, k)$, with a greater order of smoothness. Assume that the numerical integration (7.1) over the reference element has degree of precision $d \geqslant 2 r-2$. As a basis for $S_{h}$, use the normalized $B$-splines. Then $G_{h}=\Gamma_{h}$, because the numerical integration of all inner products is exact,

$$
(\varphi, \psi)_{h}=(\varphi, \psi), \quad \varphi, \psi \in S_{h} .
$$

Thus, although $Q_{h} \neq P_{h}$, the results of de Boor [10] can be used to bound $\left\|G_{h}^{-1}\right\|$, giving the result (7.8) with $\bar{n}=1$. Also, since $B$-splines are nonnegative and constitute a partition of unity, (7.6) is verified directly, with

$$
\sum_{i=1}^{N}\left|\varphi_{i}(s)\right|=1, \quad a \leqslant s \leqslant b .
$$

The assumption (l) holds easily, and this implies (7.7). Thus we also have for the general spline spaces $S_{h}=S(r, k)$ that

$$
\left\|Q_{h}\right\| \leqslant C
$$

independent of $h$, provided the degree of precision $d$ of (7.1) satisfies $d \geqslant 2 r-2$. With this, the inequalities (7.12)-(7.15) also apply to $S_{h}=S(r, k)$. In (7.15), we need $d \geqslant 2 r-1$ in order to recover the full speed of convergence of the iterated Galerkin method. This was noted previously in [12]. 
Acknowledgments. The authors would like to thank the referees for their suggestions.

Mathematics Department

University of Iowa

lowa City, Iowa 52242

1. P. M. Anselone, Collectively Compact Operator Approximation Theory, Prentice-Hall, Englewood Cliffs, N. J., 1971.

2. U. AsCHER, "Discrete least squares approximations for ordinary differential equations," SIAM J. Numer. Anal., v. 15, 1978, pp. 478-496.

3. K. AtKInson, "The numerical solution of the eigenvalue problem for compact integral operators," Trans. Amer. Math. Soc., v. 129, 1967, pp. 458-465.

4. K. AtKinson, "Convergence rates for approximate eigenvalues of compact integral operators," SIAM J. Numer. Anal., v. 12, 1975, pp. 213-222.

5. K. AtKInson, A Survey of Numerical Methods for the Solution of Fredholm Integral Equations of the Second Kind, SIAM, Philadelphia, Pa., 1976.

6. K. AtKinson, An Introduction to Numerical Analysis, Wiley, New York, 1978.

7. K. AtKinson, "Piecewise polynomial collocation for integral equations on surfaces in three dimensions," J. Integral Equations, v. 9, 1985, pp. 25-48.

8. K. AtKInson, "Solving integral equations on surfaces in space," in Constructive Methods for the Practical Treatment of Integral Equations (G. Hämmerlin and K.-H. Hoffmann, eds.), Birkhäuser Verlag, Basel, 1985, pp. 20-43.

9. K. Atkinson, I. Graham \& I. Sloan, "Piecewise continuous collocation for integral equations," SIAM J. Numer. Anal., v. 20, 1983, pp. 172-186.

10. C. DE BooR, "A bound on the $L_{\infty}$-norm of $L_{2}$-approximation by splines in terms of a global mesh ratio," Math. Comp., v. 30, 1976, pp. 765-771.

11. P. G. Ciarlet, The Finite Element Method for Elliptic Problems, North-Holland, Amsterdam, 1978.

12. G. A. Chander, Superconvergence of Numerical Solutions to Second Kind Integral Equations, Ph. D. thesis, Australian National University, Canberra, 1979.

13. G. A. Chandler, "Superconvergence for second kind integral equations," in The Application and Numerical Solution of Integral Equations (R. S. Anderssen, F. de Hoog, and M. Lukas, eds.), Sijthoff \& Noordhoff, Groningen, 1980, pp. 103-118.

14. F. Chatelin, Spectral Approximation of Linear Operators, Academic Press, New York, 1984.

15. F. Chatelin \& R. Lebbar, "Superconvergence results for the iterated projection method applied to a Fredholm integral equation of the second kind and the corresponding eigenvalue problem," J. Integral Equations, v. 6, 1984, pp. 71-91.

16. P. J. Davis \& P. Rabinowitz, Methods of Numerical Integration, 2nd ed., Academic Press, New York, 1984.

17. J. Descloux, “On finite element matrices,” SIAM. J. Numer. Anal., v. 9, 1972, pp. 260-265.

18. J. Douglas JR., TOdd Dupont \& LarS Wahlbin, "Optimal $L_{\infty}$ error estimates for Galerkin approximations to solutions of two-point boundary value problems," Math. Comp., v. 29, 1975, pp. 475-483.

19. J. Douglas JR., Todd Dupont \& Lars Wahlbin, "The stability in $L^{4}$ of the $L^{2}$-projection into finite element function spaces," Numer. Math., v. 23, 1975, pp. 193-197.

20. R. J. Herbold, M. H. Schultz \& R. S. VARGa, "The effect of quadrature errors in the numerical solution of boundary value problems by variational techniques," Aequationes Math., v. 3, 1969. pp. $247-270$.

21. G. C. Hsiao, P. Kopp \& W. L. Wendland, "A Galerkin collocation method for some integral equations of the first kind," Computing, v. 25, 1980, pp. 89-130.

22. G. C. Hsiao \& W. L. Wendland, "The Aubin-Nitsche lemma for integral equations," J. Integral Equations, v. 3, 1981, pp. 299-315.

23. S. JoE, The Numerical Solution of Second Kind Fredholm Integral Equations, Ph. D. thesis, Univ. of New South Wales, Sydney, Australia, 1985.

24. J. N. LyNESS \& D. C. JeSPERSEN, "Moderate degree symmetric quadrature rules for the triangle," $J$. Inst. Math. Appl., v. 15, 1975, pp. 19-32.

25. J. E. OsBorn, "Spectral approximation for compact operators," Math. Comp., v. 29, 1975, pp $712-725$.

26. G. R. RICHTER, "Superconvergence of piecewise polynomial Galerkin approximations for Fredholm integral equations of the second kind," Numer. Math., v. 31, 1978, pp. 63-70. 
27. L. L. Schumaker, Spline Functions: Basic Theory, Wiley, New York, 1981.

28. I. H. SLOAN, "Superconvergence and the Galerkin method for integral equations," in Treatment of Integral Equations by Numerical Methods (C. T. H. Baker and G. F. Miller, eds.), Academic Press, London, 1982, pp. 197-208.

29. I. H. SloAN \& Vidar ThOMEe, "Superconvergence of the Galerkin iterates for integral equations of the second kind," J. Integral Equations, v. 9, 1985, pp. 1-23.

30. A. SPence \& K. S. Thomas, “On superconvergence properties of Galerkin's method for compact operator equations," IMA J. Numer. Anal., v. 3, 1983, pp. 253-271.

31. A. H. Stroud, Approximate Calculation of Multiple Integrals, Prentice-Hall, Englewood Cliffs, N.J., 1971.

32. R. Whithey, "The stability of finite rank methods with applications to integral equations," SIAM J. Numer. Anal., v. 23, 1986, pp. 118-134.

Added in proof. Results related to the material of Section 2 can be found in the following reference: L. M. Delves \& J.Mohamed, Computational Methods for Integral Equations, Cambridge University Press, 1985, pp. 208-209. 


\section{Supplement to The Discrete Galerkin Method for Integral Equations}

\section{By Kendall Atkinson and Alex Bogomolny}

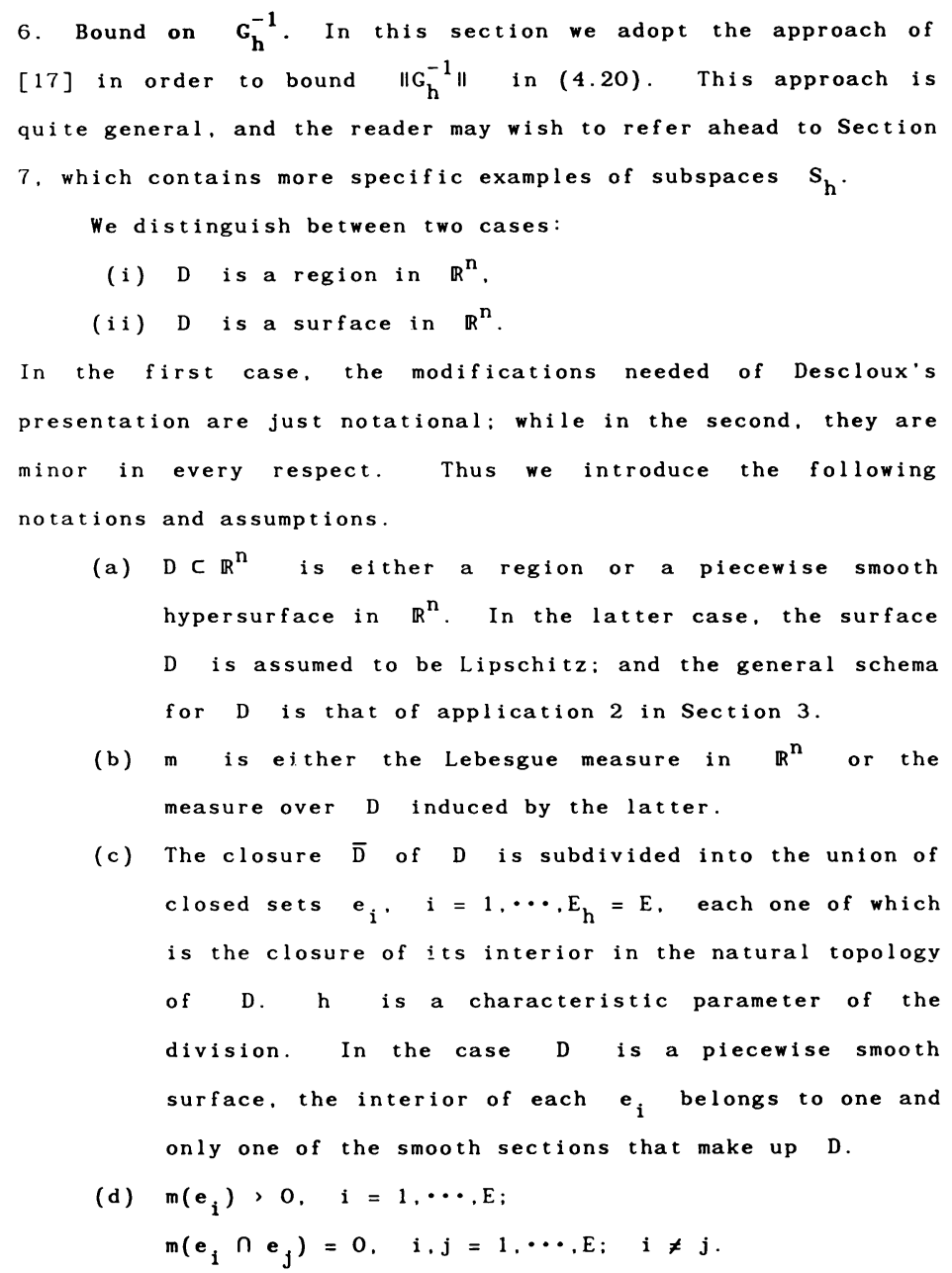

(a) $D \subset \mathbb{R}^{\mathrm{n}}$ is either a region or a piecewise smooth hypersurface in $\mathbb{R}^{n}$. In the latter case, the surface $D$ is assumed to be Lipschitz: and the general schema for $D$ is that of application 2 in Section 3.

(b) $m$ is either the Lebesgue measure in $\mathbb{R}^{n}$ or the measure over $D$ induced by the latter.

(c) The closure $\bar{D}$ of $D$ is subdivided into the union of closed sets $e_{i}, i=1, \cdots, E_{h}=E$, each one of which is the closure of its interior in the natural topology of D. $h$ is a characteristic parameter of the division. In the case $D$ is a piecewise smooth surface, the interior of each $e_{i}$ belongs to one and only one of the smooth sections that make up D.

(d) $m\left(e_{i}\right)>0, i=1, \cdots, E$; $m\left(e_{i} \cap e_{j}\right)=0, \quad i, j=1, \cdots, E ; \quad i \neq j$. 


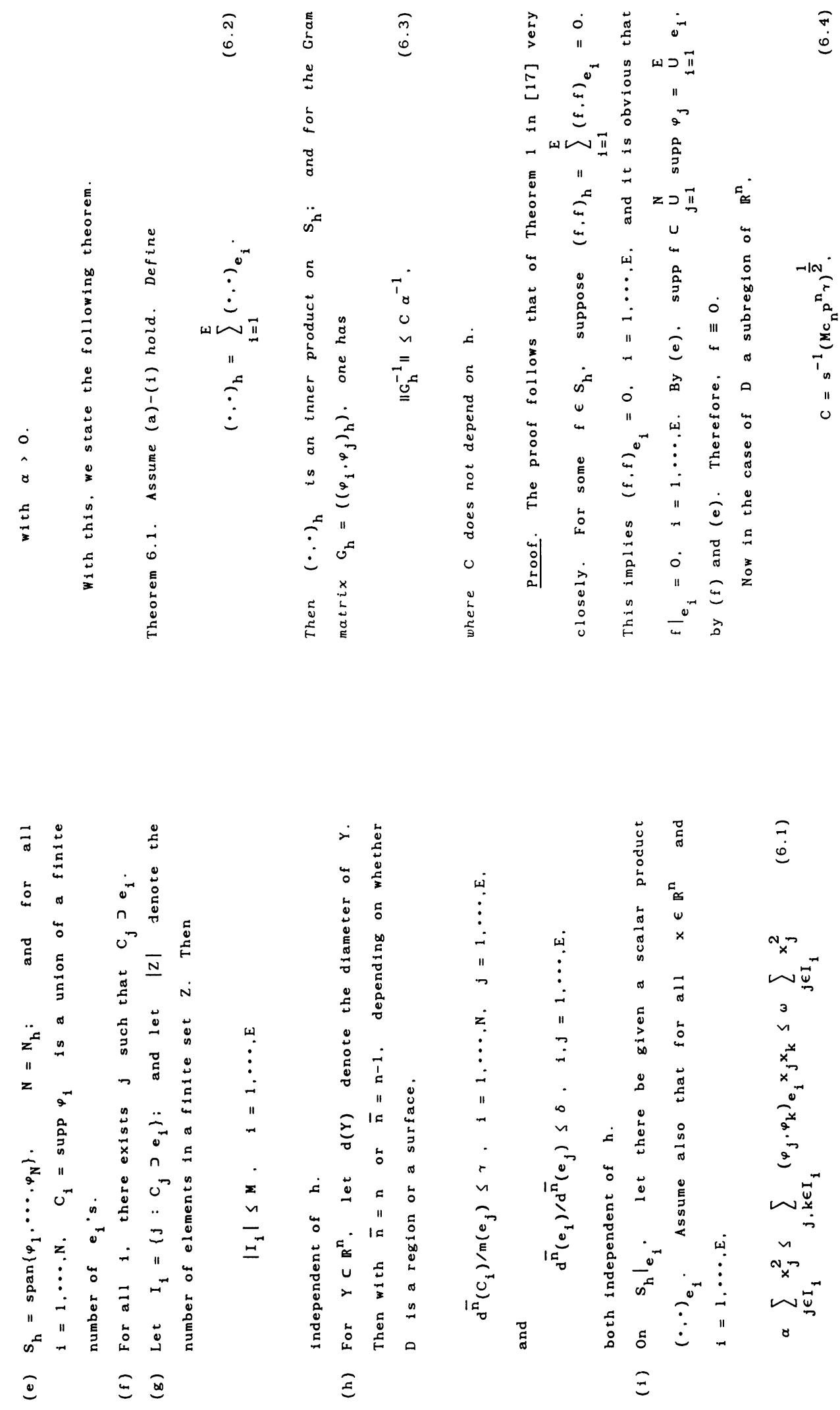




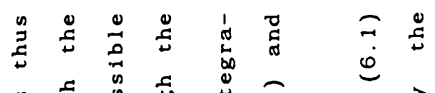

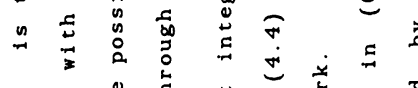

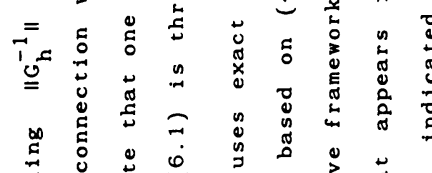

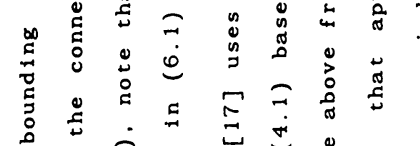

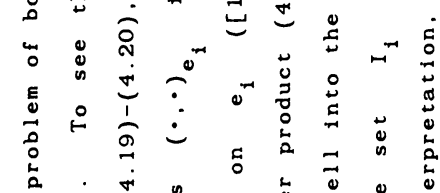

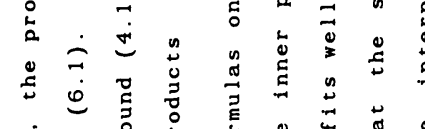

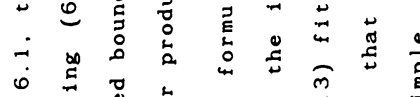

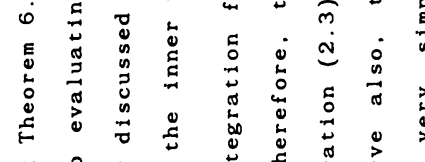

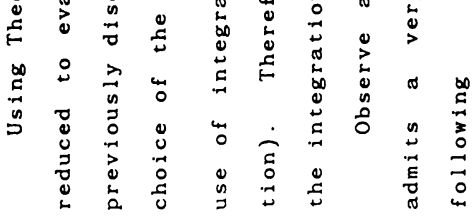

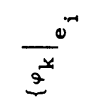

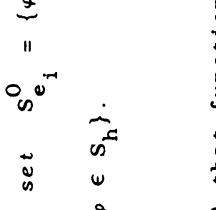

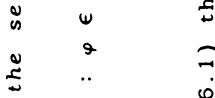

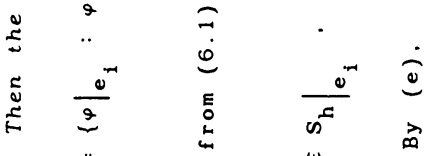

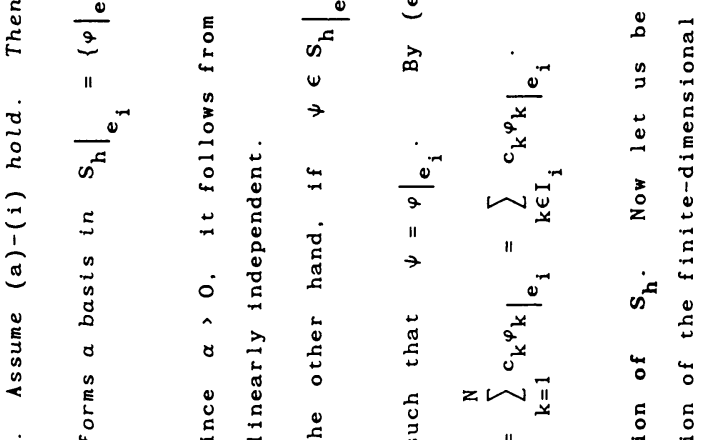

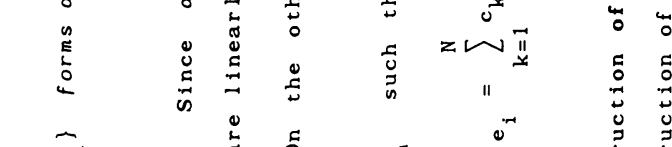

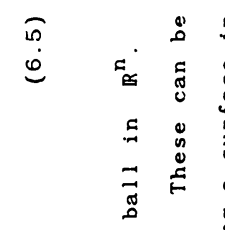

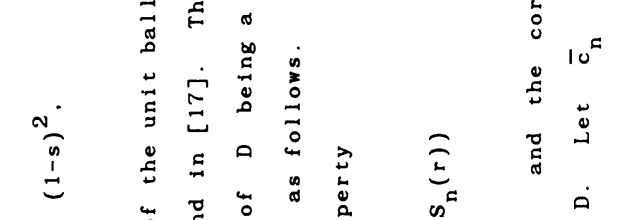

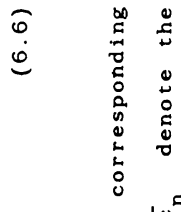

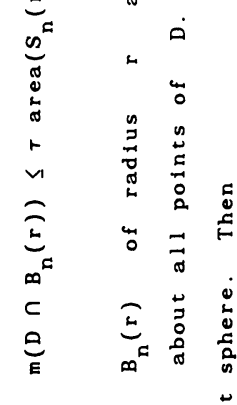

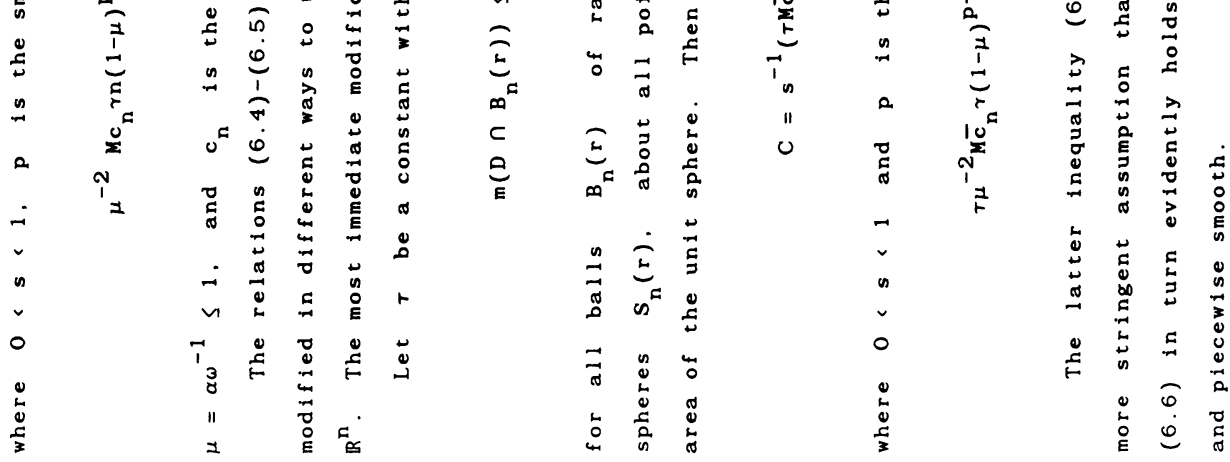

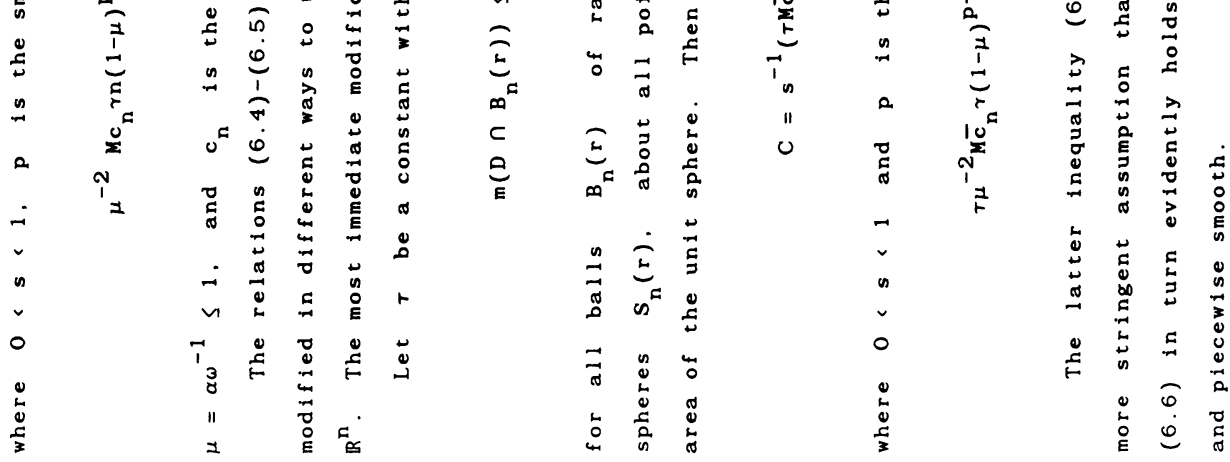

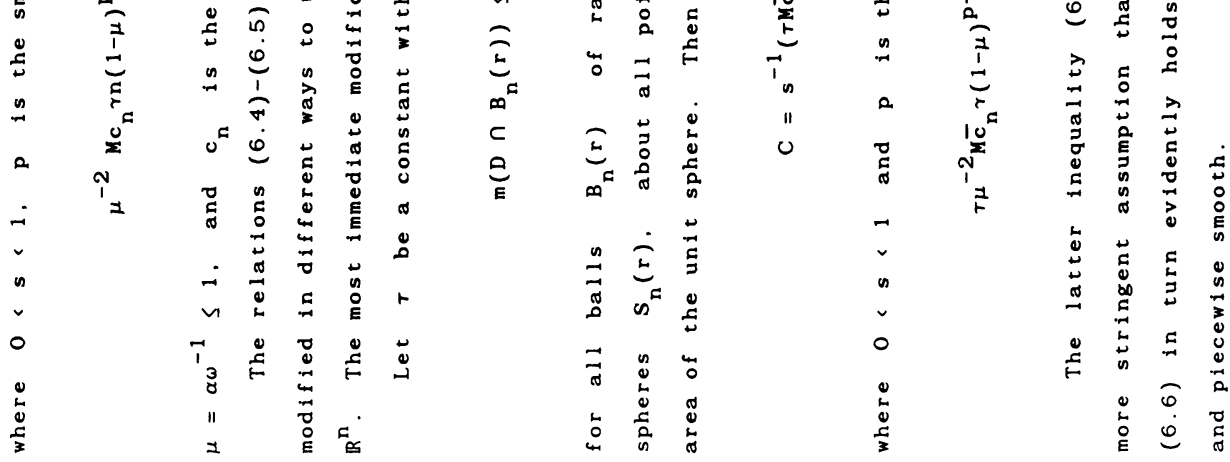

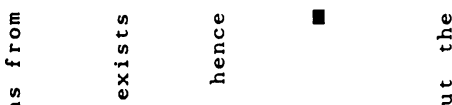

苟

$0 \quad 2$

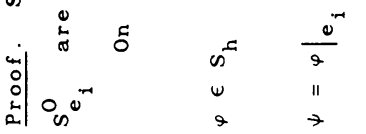

$\begin{array}{ll} & 0 \\ 0 & 0 \\ 0 & 0 \\ 0 & 0 \\ 0 & 0\end{array}$

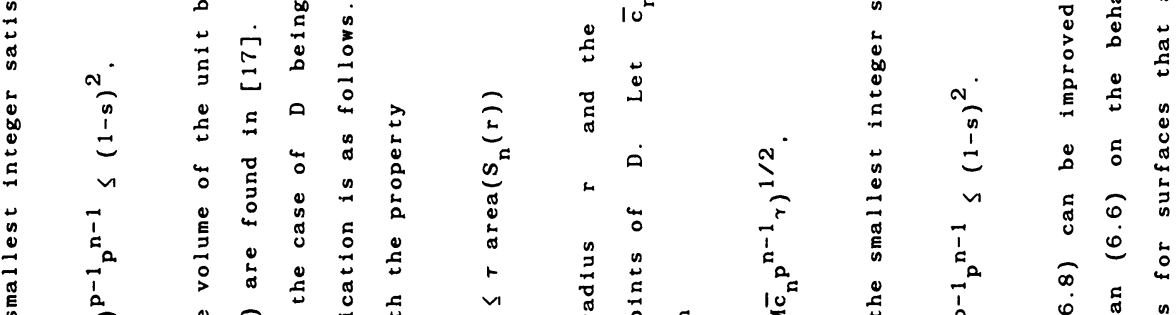

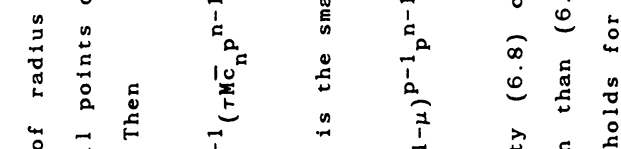



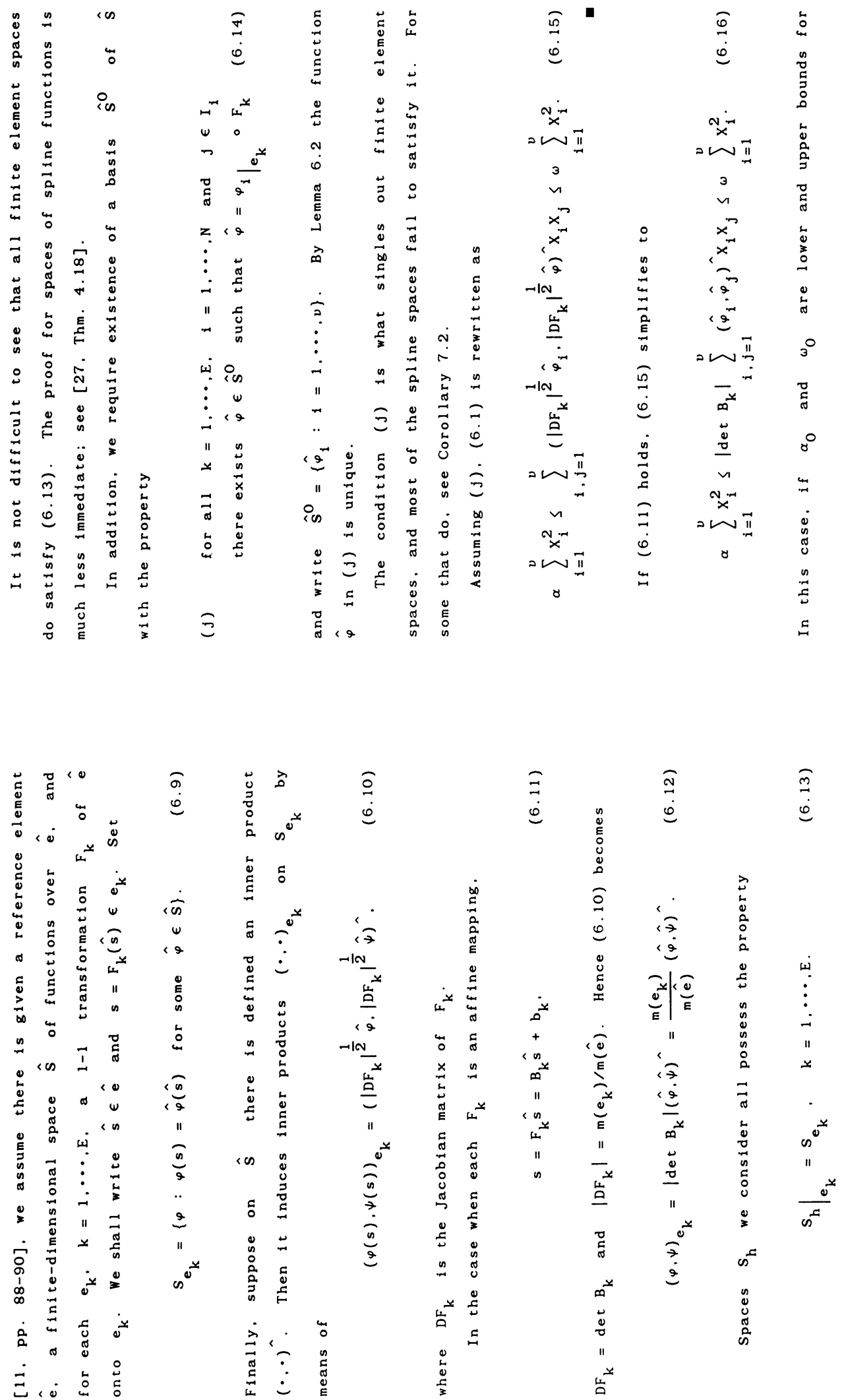

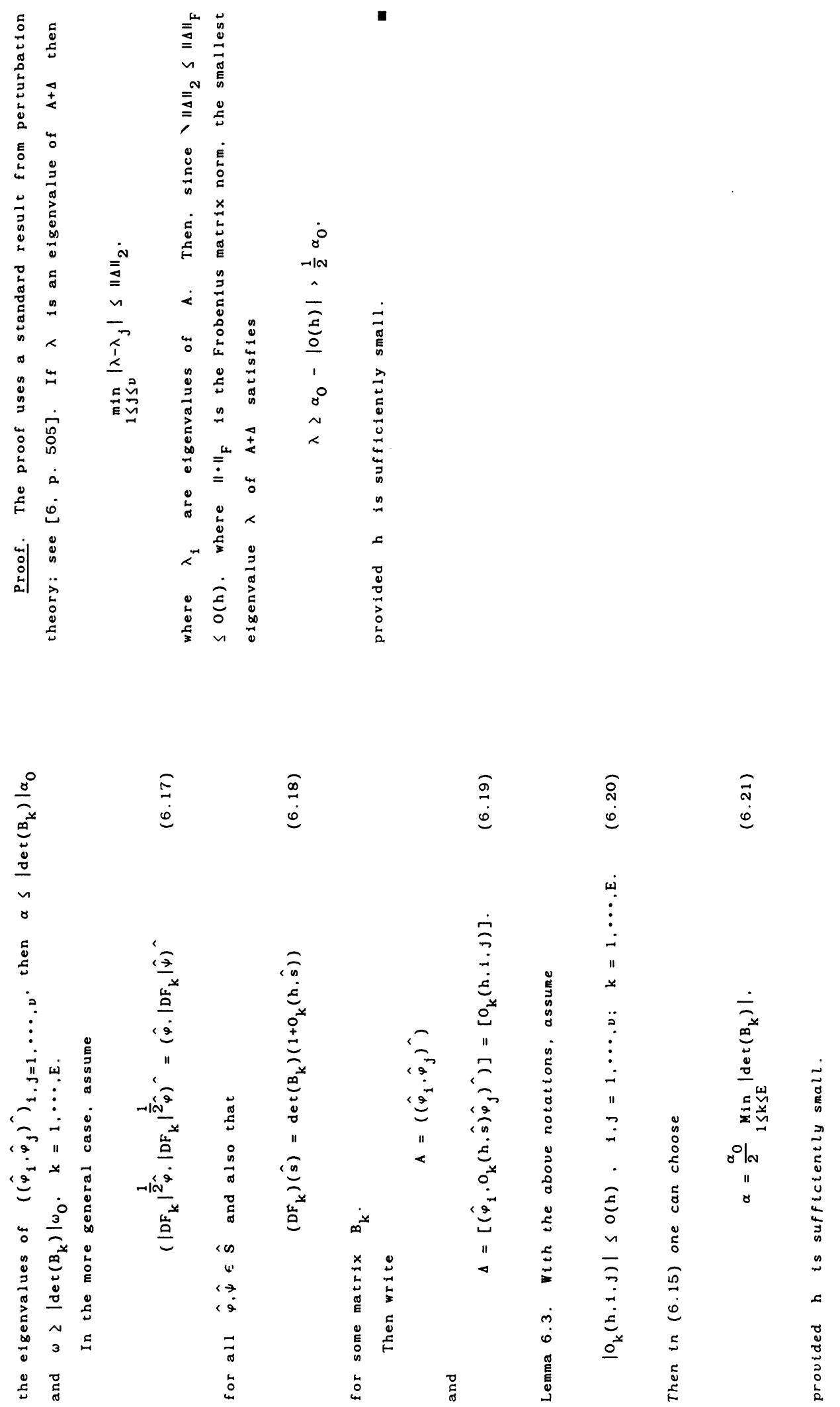